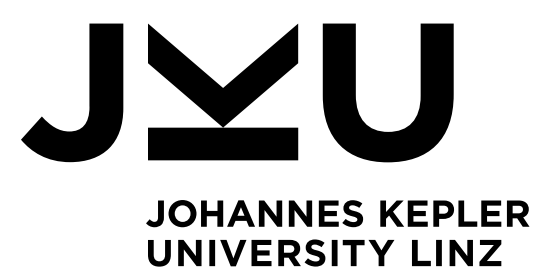

Submitted by

Manuela Pollak

Submitted at

Department of Telecooperation

\title{
E-Mail Monitoring and Management with MS Social Bots
}

Supervisor

Gabriele Anderst-Kotsis

August 2017

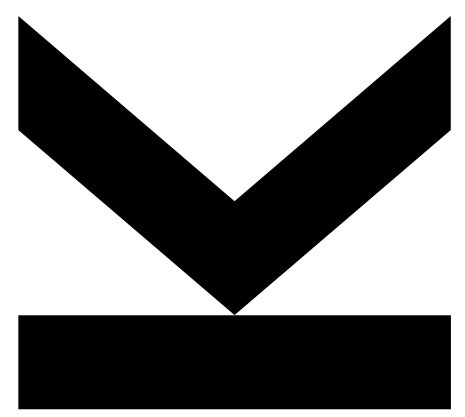

Master Thesis

to obtain the academic degree of

Diplom-Ingenieurin

in the Master's Program

066921 Computer Science (2013W) 


\section{Abstract}

Social Bots are software robots implementing algorithms that autonomously produce content and interact with users of social networks or mimic the behaviour of humans in multiple other forms of digital communication. If they act on behalf of a specific user of party in the communication, this user is sometimes called the "owner" of the bot. Social bots are typically designed to be, benevolent and even useful but some of them are built to manipulate and deceive social users.

Since spring 2016 Microsoft provides the technology Microsoft Bot Framework. With this framework developers can create own social bot applications easily in Visual Studio with the language $\mathrm{C \#}$. The bot application can be connected to various social channels like Facebook, Twitter, Microsoft Groups or an Office 365 accounts.

The developed software "Email Monitoring and Management with Microsoft Social Bots" shows how the Microsoft technology can be combined with methods from artificial intelligence to become even more productive and useful for the owner of the bot. The focus of the thesis is on proofing the technical feasibility and implementation of "intelligent" social bots with state of the art technology. 


\section{Kurzfassung}

Social Bots sind Software-Roboter deren implementierte Algorithmen autonom Inhalte produzieren und mit Nutzern sozialer Netzwerke interagieren oder das Verhalten von Menschen in mehreren anderen Formen der digitalen Kommunikation nachahmen. Wenn sie im Namen eines bestimmten Benutzers in einer Kommunikation handeln, wird dieser Benutzer manchmal auch als „Besitzer“ des Bot bezeichnet. Social Bots sind typischerweise entworfen worden um wohlwollend und sogar nützlich zu sein, aber einige von ihnen sind gebaut um Nutzern in sozialen Netzen zu manipulieren und zu täuschen.

Seit Frühjahr 2016 bietet Microsoft die Technologie Microsoft Bot Framework an. Mit diesem Framework können Entwickler eigene Social-Bot-Anwendungen in Visual Studio mit der Sprache C \# erstellen. Die Bot-Anwendung kann mit verschiedenen sozialen Kanälen wie Facebook, Twitter, Microsoft Groups oder einem Office 365 Konten verbunden werden.

Die entwickelte Software "E-Mail-Monitoring und Management mit Microsoft Social Bots" zeigt, wie die Microsoft-Technologie mit Methoden von künstlich intelligent kombiniert werden kann, um noch produktiver und nützlicher für den Besitzer des Bot zu werden. Der Fokus der Arbeit liegt dabei auf der Demonstration der technischen Machbarkeit und Implementierung von ,intelligenten“" sozialen Bots unter Verwendung von aktuell verfügbaren Technologien. 


\section{Table of Contents}

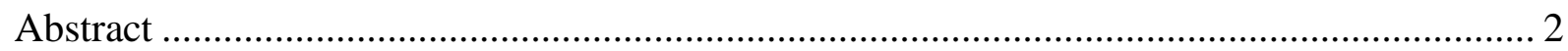

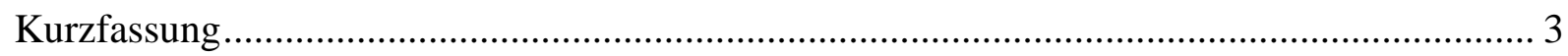

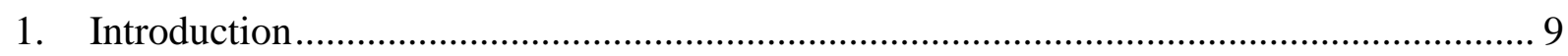

1.1. Social bots - beneficial helpers or manipulating threats .................................... 9

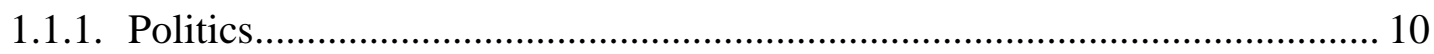

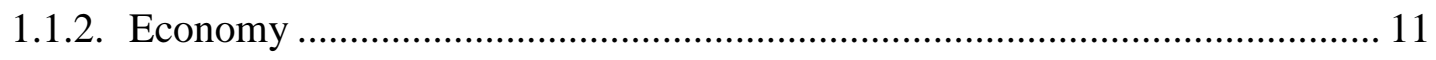

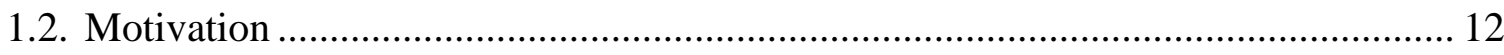

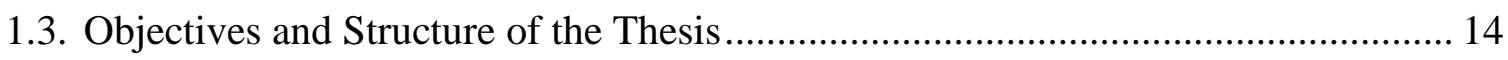

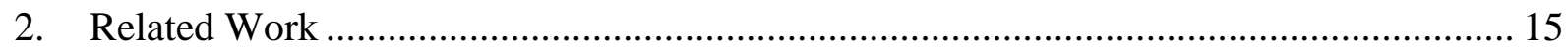

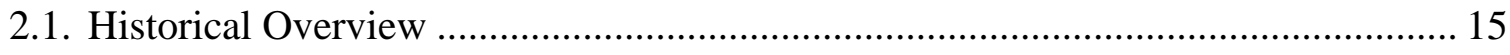

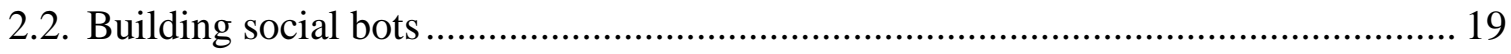

3. Introduction to social bots with Microsoft Bot Framework.......................................... 21

3.1. Microsoft Bot Framework Core Concept .............................................................. 21

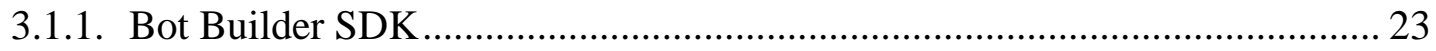

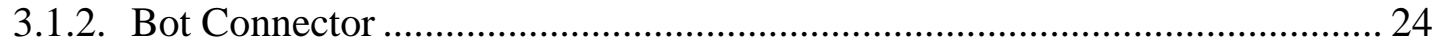

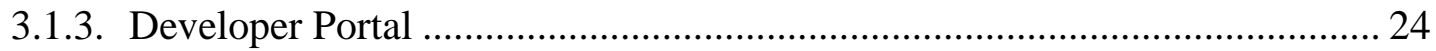

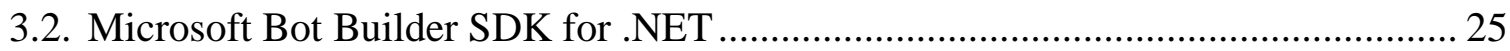

4. Selected Techniques from Artificial Intelligence ….................................................... 27

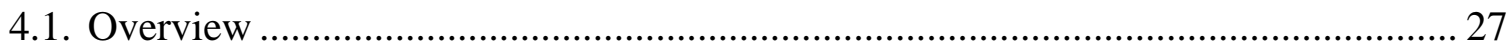

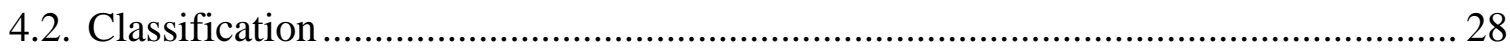

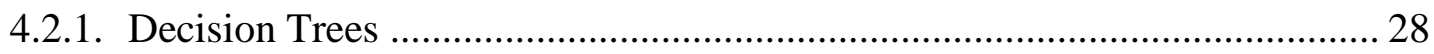

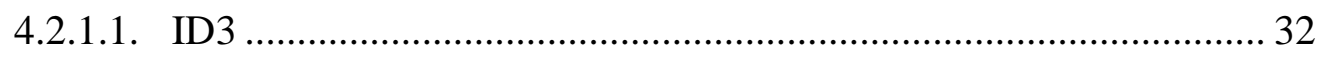

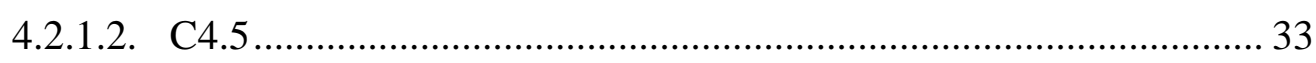

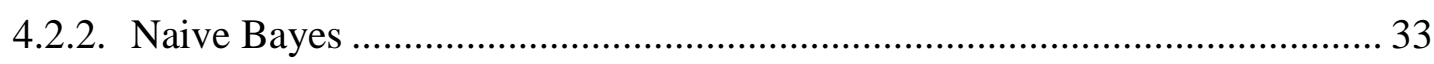


4.2.3. Support Vector Machine …..................................................................... 35

4.3. Implemented Classification ............................................................................ 36

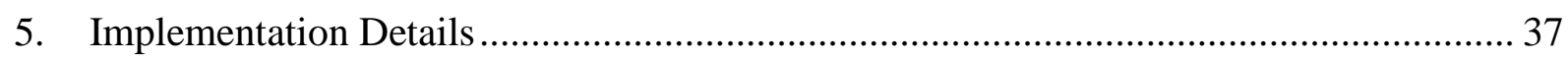

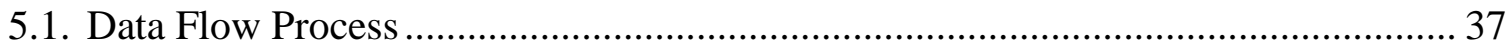

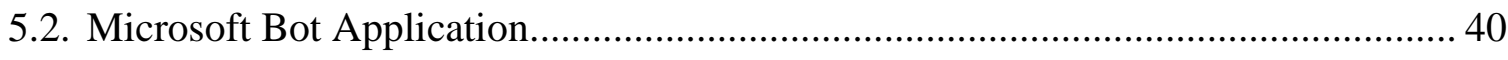

5.3. Entity Framework, Repository, Unit of Work Pattern and Microsoft SQL Database

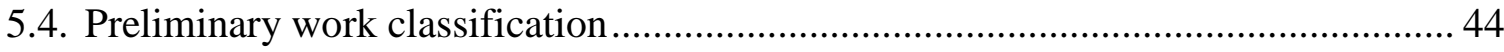

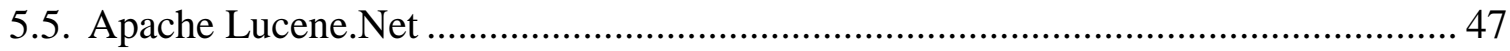

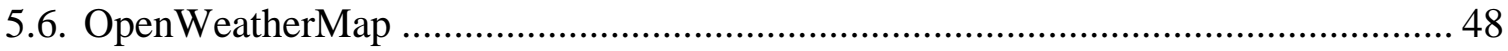

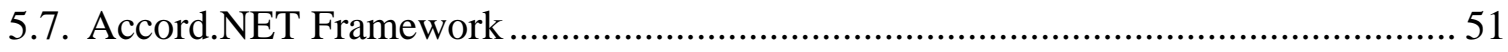

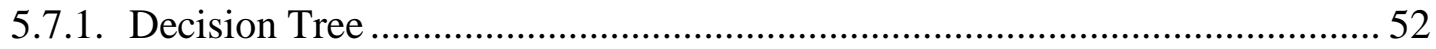

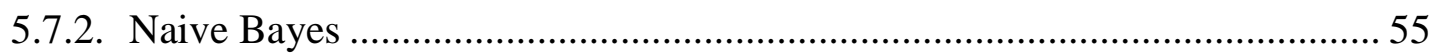

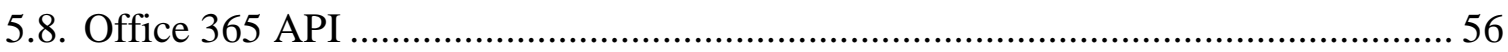

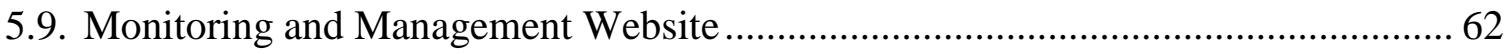

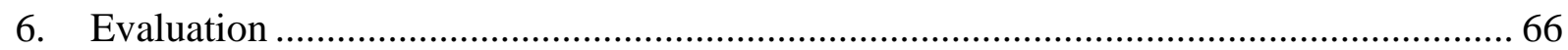

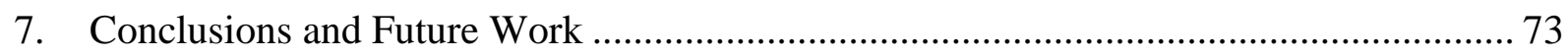

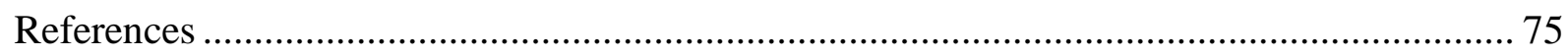

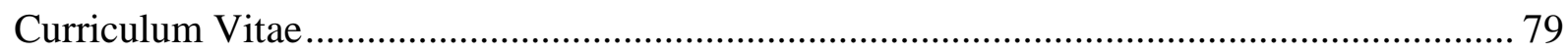

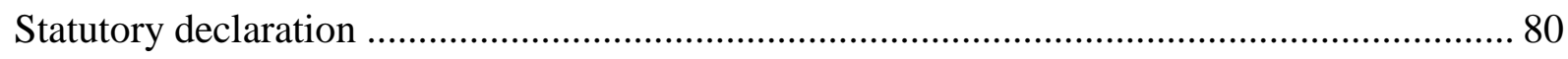

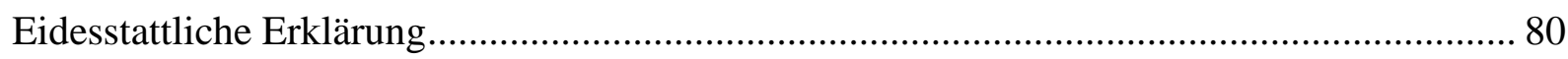




\section{List of Figure}

Figure 1: Sample Conversation with a social bot, [STA17]

Figure 2: How the Bot Framework works [PSD17].

Figure 3: BotConnector with different channel handling.

Figure 4: Simple "Echo Bot"-Post Method [Pol17]

Figure 5: Simple linear support vector machine 36

Figure 6: Mail Monitoring Bot - Flow Chart 38

Figure 7: CheckOfAppoinment method

Figure 8: Bot Framework - Post method

Figure 9: EF, Unit of Work, Repository, Database [DYK13]...

Figure 10: MS SQL - Class Diagram

Figure 11: Decisions training data

Figure 12: Decision Tree from training data 46

Figure 13: LuceneAnalyser GetKeywords Method

Figure 14: Incoming Email Body

Figure 15: Lucene.Net Keywords 48

Figure 16: GetWeather method in WeatherService class. 49

Figure 17: Deserialize XML string object to weather model class 50

Figure 18: Example OpenWeatherMap API Call 51

Figure 19: Accord.MachineLearning.DecisionTree namespace [Sou12] 52 
Figure 21: Implementation Codification from Decision Tree

Figure 22: DecisionVariable from social bot

Figure 23: Decision Tree Transform and Decision

Figure 24: Decision Tree - new decision in MS SQL Table

Figure 25: Naive Bayes Implementation 1

Figure 26: Naive Bayes Implementation 2. 56

Figure 27: Connected Services

Figure 28: AuthenticationHelper.cs

Figure 29: Office365Service GetCalendarEventsAsync method

Figure 30: Classification - free appointment check 59

Figure 31: Office365 Service AddCalendarEvent method 60

Figure 32: Office365Service GetEmailMessages method

Figure 33: Monitoring and Management Website - Login Page 62

Figure 34: Website - Home page. 63

Figure 35: Website - Office 365 calendar events view page

Figure 36: Website - Decisions -Training data view page 64

Figure 37: Website - Create new decision view..... 64

Figure 38: Website - Shortcut Classification results 65 


\section{List of Tables}

Table 1: Evaluation - confusion matrix

Table 2: Evaluation - Sample data with Classification results

.68

Table 3: Evaluation - confusion matrix result Decision Treet.

Table 4: Evaluation - confusion matrix result Naive Bayes.

Table 5: Evaluation - Classification measures 


\section{Introduction}

\subsection{Social bots - beneficial helpers or manipulating threats}

Social bots are a kind of software robots with algorithms designed to hold a conversation with a human in social networks. Social bots mimic humans and humans' behaviour and they are often useful but some are created to manipulate, spread misinformation or harm others [Fer15]. They can also act as chatbots and communicate with users synchronously.

Today there are more than a million people communicating in social networks like Facebook and Twitter. These social networks can be used for many different reasons, such as private statements, sharing photos, marketing, advice or as a political statement platform. It didn't take long for organisations to realize that such social networks are perfect platforms to influence people. Social bots are in the beginning to spread across social media. Access to social bots is becoming easier and more companies and political parties are discovering the power of such technology.

Social bots are interacting and working independently, make their own decisions based on their human environment and try not to attract attention by unusual behaviour patterns. Social networks are their main target for reaching many people with very little effort. By means of word-of-mouth advertising something should be communicated, which appeals to a lot of people, give positive response and share the message.

However, social bots can only be successful if many people accept their friend request. And even more successfully are they if the people provide them their user profile. Canadian scientists investigate the infiltration of social bots on the social media network Facebook by connecting them with real users. The success rate up to $80 \%$ shows how careless many users are with their account [BMB11]. The target of many social bots is to collect user data like

- gender,

- birth date,

- married to,

- worked at, 
- current city,

- home city or

- even email addresses.

These collected personal data is then sold to third parties like private companies to take further advantage of that.

Meanwhile, there are already services to find social bots also called fake accounts in the social networks. The best-known service for Twitter account is BotOrNot ${ }^{1}$. This online service is a Twitter bot detector that is able to spot fake followers. Therefore, the website uses the Twitter's Rest $^{2}$ API to obtain the account's recent history, including recent tweets, available meta-data and computes a bot-likelihood score by using a classification algorithm [DVF16].

There are many intended uses for social bots. The greatest conceivable fields of application are in politics and economy, since money and power play a very important role there.

\subsubsection{Politics}

Over the last several years the number of political parties, militaries and government-contracted firms which use the digital power of social bots that has been rising significantly. The social bots goal is to manipulate public opinions and disrupt organizational communications.

Bots are used to share or retweet content from political parties on one hand. So, in Venezuela where social bots or also called political bots have a small strategic role in political conversations. Four computer scientists have analysed six active twitter accounts of six politicians. They found out that less than 10 percent of retweets of that accounts are from botrelated platforms [FHM15].

On the other hand, social bots are used as tool for spreading disinformation and attacking users. In the US, social bots have become artificial election campaign assistant that support political

\footnotetext{
${ }^{1} \mathrm{http} / / /$ botornot.co/

${ }^{2}$ Rest $=$ Representational state transfer
} 
parties. At the U.S. Presidential election in 2016 both candidates, Hilary Clinton and Donald Trump, deployed their artificial assistant on Facebook and Twitter. Alessandro Bessi and Emilio Ferrara investigated how many social bots affect the political discussion around the 2016 U.S. Presidential election. They estimated that about 400,000 bots are engaged in the political discussion about the Presidential election. Those social bots are responsible for about 3.8 million tweets, which should be about one-fifth of the entire conversation [BES16].

Social bots influence the mood on the internet by automatically sending thousands of tweets in just a few seconds and the bots developer often remain in the dark.

\subsubsection{Economy}

Just as in the politics, the use of social bots in the privately owned market is ongoing. Not only companies are buying private data collected by social bots for marketing purposes, as mentioned above. Companies with a high fan rate on their social media channel have a higher head value because they can reach a higher number of more people. To quickly achieve a large number of followers and fans, many companies already buy fake profiles. However, these profiles are not made and used by humans. They are used and utilized by bots. The Dutchman Lennart V. is a social bot developer and he is a specialist on developing fake-accounts. He does not sell these created accounts to individual companies but rather at transactors, and he offers the packages starting from 10,000 accounts onwards [BUE16].

So far, the entrance for chat bots in the economy was still high. Without a team of developers, bots could not be implemented. Now some startups uses the opportunity. One is the company Chatfuel $^{3}$. They have developed an online tool where companies could create their own chat bots without writing a single line of code. Chatfuel offers this option two social media platforms, these two are Telegram messenger and Facebook Messenger. Adidas ${ }^{4}$ is one of the famous companies that provides a Facebook chatbot of Chatfuel. Adidas focuses on its female customers and presents its new studio LDN. In addition to general information on the studio,

\footnotetext{
${ }^{3}$ https://chatfuel.com/

${ }^{4}$ https://m.me/adidaswomenuk/
} 
the bot provides an interactive booking service for weekly free-to-access fitness sessions. The goal is to enhance the brand.

There are many advantages of using chatbots for customer relation management (CRM) in a company, just a few are highlighted now:

- Chatbots are available $24 / 7$

- Bots can replace call centers

- Chatbots allow direct interaction with clients, ease way to collect information about customers' needs and interests.

- Deliver targeted content based on a user's feedback.

Chatbots can be used in many beneficial ways. They can be used as virtual consultants (for example, for clothing, travel, restaurants), virtual assistants (requests from authorities, universities), intelligent search engines (by requesting preferences), or as a virtual call center.

The Domino's Pizza ${ }^{5}$ chatbot is a good example of customer service. With the chatbot customers are able to order directly and track their order, this option is a simple, effective customer service tool.

\subsection{Motivation}

During my practicum project, I got familiar with the Microsoft Bot Framework. With this Bot Framework developing social bots has become very easy and the question, whether social bots can be used for private applications with benefits, comes up. The Bot Framework allows an integration with Office 365 Email and so incoming Emails can be managed on this way. Email clients like outlook or -Gmail webclient already have many useful possibilities for integrating functions, rules or filters to deal better with the email masses. Rules or filters are possible for:

- Automated move messages to folder,

\footnotetext{
${ }^{5}$ https://www.messenger.com/t/dominos/
} 
- Forward email to someone,

- Start an application/script,

- Play a sound by incoming email

- Automated answer to sender

- Delete message

Setting those rules or filters have to be done manually and therefore, the clients are very simple, rigid programmed and also not expandable.

Is there a possibility where social bots can help us with our daily Email flood?

The answer is yes. The topic of this master thesis is about how bots can deal with appointment requests out of incoming emails. The most important question is, is there a possibility to combine the Microsoft Bot Framework with Artificial intelligence (AI) and which kind of these AI methods are needed for the decision-making process?

So the main purpose of the implemented bot application of "Email Monitoring and Management with Microsoft Social Bot“ is filtering appointment requests out of incoming Emails and to analyse them with AI. The core concept is:

- Proof the request text with a text-analytic service,

- Check Office 365 calendar for free appointment,

- Decision-making process for acceptance of appointments,

- If decision-making process succeeded then create a new calendar event in the Office 365 calendar and

- Automatic answering of incoming appointment requests.

For the decision-making process one possible method of the AI is classification. In classification a general structure, from existing data, is to find and so a conclusion can be drawn to new data. Four possibilities of classification are shown and two of them are integrated into the social bot. 


\subsection{Objectives and Structure of the Thesis}

This master thesis is organized as follows:

This current chapter gives an overview of the master thesis and its central questions, as well as the structure.

In chapter two related work regarding artificial intelligence and social bots is reviewed.

Chapter three gives an overview about how social bots are implemented using the Microsoft Bot Framework. The main features of this framework which are necessary to understand the implementation part of this thesis are explained.

Basic concepts from artificial intelligence are introduced in chapter four with a focus on classification techniques. Specifically, Decision Trees, Naïve Bayes and Supported Vector Machines are described in detail.

Chapter five describes the implementation of the social bot with artificial intelligence. The process sequence is described and subsequently all implemented technologies are specified.

Chapter six deals with the evaluation of the performance of the two implemented decisionmaking techniques (Decision Tree and Naïve Bayes). A set of test emails is used in the evaluation.

The main results are summarized in the conclusions in chapter seven and an outlook on future work is given. 


\section{Related Work}

In this chapter, a historical overview to understand the evolution of the field, followed by a survey of possible implementation methods and techniques is given.

\subsection{Historical Overview}

Chatbots are very trending right now but the idea isn't new. The history of the fascinating chatbots starts way earlier, they have been around since more than 60 years.

The pioneering British computer scientist Alan Turing started visioning of a conversation between machine and humans in 1950. In his famous article "Computing Machinery and Intelligence" Alan Turing proposed the Turing test which acts as a criterion for intelligence. The criterion depends on the ability of a computer program to act as a human and its behaviour in a real-time conversation with a human chat partner. The goal is convincing the human partner into thinking that the human is chatting with another real person. Therefore, the Turing test makes it necessary for the chatbot to understand human language and respond in the most human way with his language. This results in three main components:

- For understanding the input conversation, a parser is needed

- A knowledge process for understanding the context and other details

- For replying to the chat partner, a reply natural language generator is needed.

Since the publication of the Turing test the dream of designing such a computer algorithm expedited artificial intelligence research for decades. Different approaches of chatbot with different personality attributes or purposes have been implemented.

One of the most famous chatbot is ELIZA. ELIZA was created in 1966 by Joseph Weizenbaum to demonstrate certain kinds of natural language conversation between human and machine. Input sentences are analysed based on decomposition rules which are triggered by key words appearing in the input text. Responses are generated by reassembly rules associated with selected decomposition rules. This directive on how to interact where provided by scripts. ELIZA is a chatbot which just is listening to its partner and never speaks about itself. This 
works with selective questions like "Does it please you to believe that ...?" additional the question of the chat partner. The most famous script which exists simulates a Rogerian ${ }^{6}$ psychotherapist [Wei66]. ELIZA appeared to pass the Turing test.

The first chatbot which passed the Turing test was PARRY. PARRY was developed to simulate a schizophrenic by reproducing the logic of paranoid human thoughts through an algorithm. The chatbot was written in 1972 by psychiatrist Kenneth Colby and includes more tricks than ELIZA used to [Mau94]:

- Admitted ignorance: PARRY is able to say, "I don't know" in response to a question.

- Changing conversation level: by asking questions.

- Includes little stories and build them into the conversation.

- Introducing new topics: PARRY could introduce new topics in imitation of paranoid behaviour.

Colby conducted blind tests with experienced psychiatrists who tested in a machine-mediated interviewing environment corresponded with real-life paranoid patients and PARRY through teletype. Other 33 psychiatrists were shown transcripts of the conversation and then they all were asked to identify which of the patients were human and which were a computer program. Only 48 percent made the correct identification [CHW72].

The British computer scientist Rollo Carpenter launched in 1989 the chatbot Jabberwacky and since 1997 the chatbot has been running on the web ${ }^{7}$. It aims to simulate a natural, entertaining and humorous chat with a human partner. Therefore, models for human learning behaviour are developed, such as children's languages, rules and individual facts in their specific context. The chatbot uses only previously learned material, that means it accesses a database that contains all the user's previous input, for each additional input, a suitable response is calculated from this fundus using complex technique of pattern recognition. Since Jabberwacky was available on the Internet the user's responses have risen from 20,000 to over 10 million (2008). The

\footnotetext{
${ }^{6}$ https://en.wikipedia.org/wiki/Person-centered_therapy

${ }^{7}$ http://www.jabberwacky.com/
} 
chatbot won the bronze medal Loebner Prize ${ }^{8}$ in 2005 as "George" and 2006 as "Joan" [DAB08].

In the early 1990s Dr. Sbaitso was the first MS-DOS program, based on an algorithm similar to that of ELIZA and was manufactured by Creative Labs. Sbaitso, acronym for Sound Blaster Artificial Intelligence Text to Speech Operator, imitated the behaviour of a psychotherapist and was equipped with various sound cards for repeating text out loud for all was typed after the word "say". Demonstrating the use of sound cards and the ability to use spoken human language was the intention of Dr. Sbaitso. The communication itself wasn't in the focus of this work. [Der10].

One of the most famous chatterbot is A.L.I.C.E. (Artificial Linguistic Internet Computer Entity). Richard Wallace created the natural language processing chatterbot in 1995 and it was rewritten in Java in 1998. A.L.I.C.E. was inspired by Weizenbaum's classical ELIZA program but it was significantly more complex. The bot generated responses by pattern matching inputs against < pattern> (input)<template>(output) pairs stored in documents in a knowledge base. These documents were written in Artificial Intelligence Markup Language (AIML) which is an extension of XML [Wal17]. A.L.I.C.E. won the Loebner Prize three times but so far, the chatbot was not able to pass the Turing test [Der10].

In 2001 the company ActiveBuddy, with his co-founder Peter Levitan, launched SmarterChild, a chatbot for AOL and MSN Messenger as well as for SMS. It was possible for users to send a request on various topics, such as weather, stock information, movie times, yellow pages listings, detailed sports or even news and the bot responded within a few seconds. In many ways, SmarterChild was a precursor to nowadays Samsung's S Voice or to Apple's Siri. SmarterChild was already very successful at the time with a considerable user number of more than 30 million. The company was sold to Microsoft in 2007 [New 16].

In 2006 the first famous question answering computer system was built by IBM DeepQA and is called Watson. Watson has been developed to successfullycompete against top human players in Jeopardy! The program shows the interaction of

\footnotetext{
${ }^{8}$ http://www.loebner.net/Prizef/loebner-prize.html
} 
- Natural Language Processing (NLP),

- Information Retrieval (IR),

- Machine Learning (ML),

- Knowledge Representation and Reasoning (KR\&R)

and delivers high degrees of precision with a tree-second response time [Fer10]. Since February 2010, Watson was able to beat humans Jeopardy!. IBM first performed an exercise session in a conference room which mimics the situation at Jeopardy. The computer system on which Watson was running provided the rate instructions electronically and was able to operate the buzzer and to give the answers in Jeopardy's own question format with an electronic voice. Finally, Watson joined Jeopardy in three broadcasts in February 2011 against two former champions. Nowadays Watson's API is available to software engineers to enable them to build apps, social bots and services that are empowered with Watson's capabilities.

Another type of social bots is the class of personal assistants, software like Apple Siri ${ }^{9}$. This originates from the project CALO, Cognitive Assistant that Learns and Organized, of the US military which was issued by DARPA's (Defense Advanced Research Projects Agency) PAL (Personalized Assistant that Learns) program in 2003 and Apple had taken over the company Siri Inc. in 2010. The software is an intelligent personal assistant and knowledge navigator that uses a natural language UI. Just by voice commands almost any feature of the mobile phone can be used. It paved the way for all AI bots [GeB16].

Google Now, developed by Google Inc. in 2012, is a bot which provides voice recognition software for processing natural speech. As a competing product to Apple's Siri Google Now was first available in Android 4.1 on Galaxy Nexus. In summer 2017 Google Now will be replaced by Google Assistant, who will be able to get to know the user and the service will be able to respond to past questions and so answer further question [SGS17].

Alexa, another personal assistant software was developed by Amazon in 2015. The inspiration was cloud-based AI which makes it easy for users to walk around the house and get some action performed or get some type of question answered. Amazone was opening the Alexa Skills Kit

\footnotetext{
${ }^{9}$ https://www.apple.com/de/ios/siri/
} 
SDK for Node.js to third-party developers for developing new skills and offering them on Amazon marketplace.

Microsoft's version of personal assistance calls Cortana and was developed in 2015. Cortana is a software that recognizes voice commands and responds in addition to making recommendations and setting reminders.

Back to social bots. In 2016 Microsoft released an intelligent social chatbot called Tay, on Twitter Tay can be found under the pofile name @ TayandYou. It is a self-learning social chat program with a female avatar on various social media platforms especially on Twitter. Microsoft promised:” The more you speak, the smarter will Tay be". The AI behind Tay is to learn from people by training with texts and questions. After some hours Tay could tell jokes, comment photos, play games, tell stories, draw Hitlers' comparisons and deny the Holocaust. User teach the bot racism and after 16 hours of chat, the experiment had to be terminated [Per16].

\subsection{Building social bots}

Social bots can be implemented in the most common programming languages like Java, C++, Pascal and in spring 2016 Microsoft released their open source technology Microsoft Bot Framework. This open source toolkit enables engineers to build their own bots in the programming language C\# [Vin16]. In my practicum project from 2017 an overview of implementing a social bot with Microsoft Bot Framework including the integration of Facebook, Skype and Email Channel is given [Pol17].

Another, much simpler, way to develop social bots is to use automated tools for building bots. For most of these chatbot creator applications no programming knowledge is required. The most popular bot creators are:

- Chatfuel: Chatfuel is focused on Facebook Messenger and no coding skills are required to get started. It contains a minimalistic AI and additional feature in chatfuel is the easy navigation steps for guiding the consumers. 
- Botsify ${ }^{10}$ : Botsify is mainly for Facebook Messenger and has a simple to use interface to build a chatbot without any coding required.

- Beep Boop ${ }^{11}$ : Beep Boop is a developer platform for building Slack integrations. Hosting, sharing and deploying Slack integrations can be done very quickly as it is possible to build own customer support tools.

- API.ai ${ }^{12}$ : This is a great platform for developing chatbots with advanced service like AI support, intuitive interface and many integration possibilities like Action on Google, Amazon Alexa, Microsoft Cortana, Facebook Messenger, Kik, Cisco Spark, and many more. Unfortunately, API.ai does not support an integration with Microsoft Office 365 and therefore, due to its complexity and temporal expenditure it's not possible to use it for this master thesis.

Emergence of cloud solutions for cognitive computing opened a whole new perspective for developing. With those services developers can make their applications more intelligent, engaging and discoverable. Cognitive services can be used for text analysis but also for picture and video analysis and also they make bot experiences more personal and productively by progressively seeing, hearing and speaking. Most popular cognitive services are:

- Microsoft cognitive services ${ }^{13}$ : Provides services like Computer Vision, Content Moderator, Speaker Recognition, Bing Spell Check and Language Understanding.

- Google Cloud Platform ${ }^{14}$ : Provides services like Compute, Storage \& Database, Networking, Big Data, Internet of Things, Machine Learning and Identity \& Security.

\footnotetext{
${ }^{10}$ https://botsify.com/

${ }^{11}$ https://beepboophq.com/

${ }^{12} \mathrm{https} / / /$ api.ai/

${ }^{13}$ https://azure.microsoft.com/en-us/services/cognitive-services/

${ }^{14}$ https://cloud.google.com/
} 
- IBM Watson ${ }^{15}$ : Provides a variety of services for developing cognitive application with Alchemy Language application.

\section{Introduction to social bots with Microsoft Bot Framework}

At the spring-conference in 2016 Microsoft presented their special developer framework Microsoft Bot Framework. This framework is a powerful tool and that allows developers to build and connect intelligent bots to the world. Conversations can appear in many different forms and over many different channels like communication via Kik, Skype, Slack, Office365 Mail or other popular services like Facebook or Twitter [STO16].

In this chapter, an introduction about the Microsoft Bot Framework is given by highlighting the main features which are necessary to understand the implementation part of this thesis.

\subsection{Microsoft Bot Framework Core Concept}

The Microsoft Bot Framework is a platform for building, connecting, testing and deploying social bots. Building with this framework can be done in different ways and for different languages with support for .NET, Node.js and REST.

Social bots built with the Bot Framework can communicate conversationally with:

- simple text strings,

- more complex rich cards that contain text, image and action buttons, and

- natural language interactions like guided interactions.

In Figure 1 a version of a social bot is shown which is used to take care of reservations in a salon appointment. The task of this bot is to understand the users intend, presenting appointment

\footnotetext{
${ }^{15}$ https://www.ibm.com/watson/
} 
options using action buttons, displaying the user's selection when they tap an appointment and sending a thumbnail card that contains the appointment information [STA17].

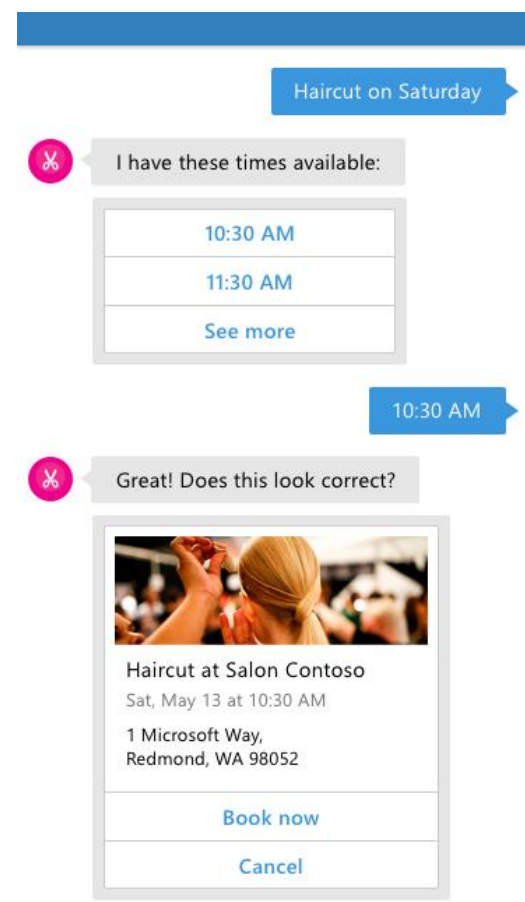

Figure 1: Sample Conversation with a social bot, [STA17]

In order to build intelligent bots, developers can add artificial intelligence to the bot with Cognitive Services. After building the bot in the Integrated Development Environment (short IDE) the bot must be registered on the Developer Portal where the bot can be connected to users through the various channel. To publish the bot, it is necessary to deploy the code in to a cloud service such as Microsoft Azure [PSD17]. 
Figure 2 gives an overview how the Bot Framework works.

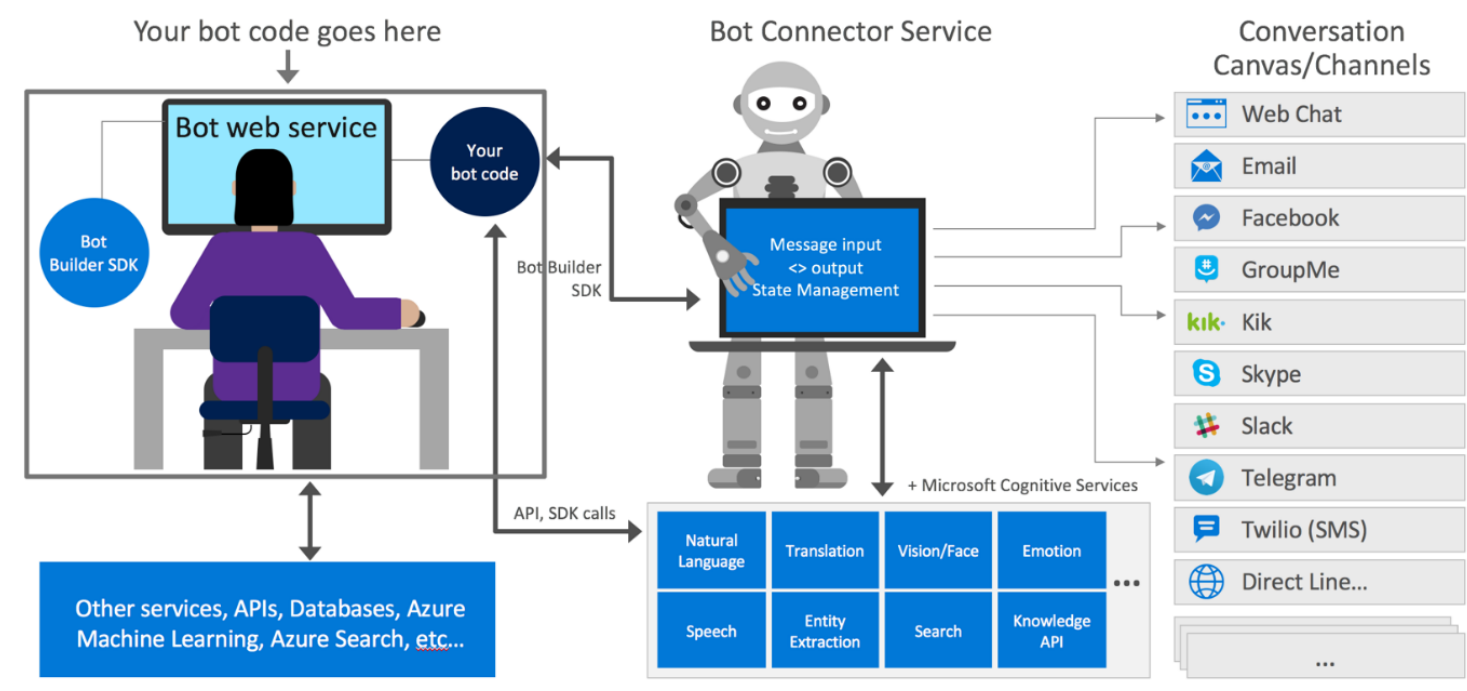

Figure 2: How the Bot Framework works [PSD17]

The Bot Framework includes the Bot Builder SDK, Bot Connector and the Developer Portal.

\subsubsection{Bot Builder SDK}

The Bot Builder SDK is open source, available on GitHub ${ }^{16}$ and provides rich and full-featured SDK's for the .NET and Node.js platforms. The SDKs provides with features like modelling conversations, rich attachments and the understanding of different languages with smart cognitive services for a simpler interaction between bots and users [Pol17].

There are three ways to build the conversation interface:

- Dialog

- Dialog models a conversation and manages conversation flows and contains the stack of dialogs that are active in the conversation at any point in time.

- FormFlows

\footnotetext{
${ }^{16}$ https://github.com/
} 
- FormFlow can generate the dialogs to manage a guided conversation. The user is moving from question to question automatically.

- Calling

- Calling allows audio calls with Skype and uses the Interactive Voice Response (IVR) technology to automate common tasks for incoming user calls.

\subsubsection{Bot Connector}

The Bot Connector is a service that connects a bot to one or more communication channels. This service allows communication without manually designing special messages for each channel. Provided channels are Skype, Slack, Office 365 mail, Facebook Messenger, Kik and many more.

The connector uses the object activity to exchange information between bot and channel. This object presents the JSON data and for sending and receiving this activity objects the connector uses Http POST and GET requests. The activity object has various types and only the type message is visible for the user. The other types serve the developer as notification and they are called ConversationUpdate, ContactRelationUpdate, Typing or DeletedUserData [Pol17].

State management, message routing and storage service are the main features of the Bot Connector.

\subsubsection{Developer Portal}

On the Developer Portal bots can be registered, connected and managed. After the registration channels can be set to each bot separately. This is a simple way to develop one chat bot for many different channels without changing the code for each of it.

The property ChannelId in the activity object hands back the information about the received message. Figure 3 shows the handling possibility with the property ChannelId. According to the channel query, this can be handled individually and after that the method ReplayToActivity() from the Bot Connector send the message back to the user. 
The Developer Portal also provides diagnostic tools and a web chat control which allows to test the bot or to embed it on a web page.

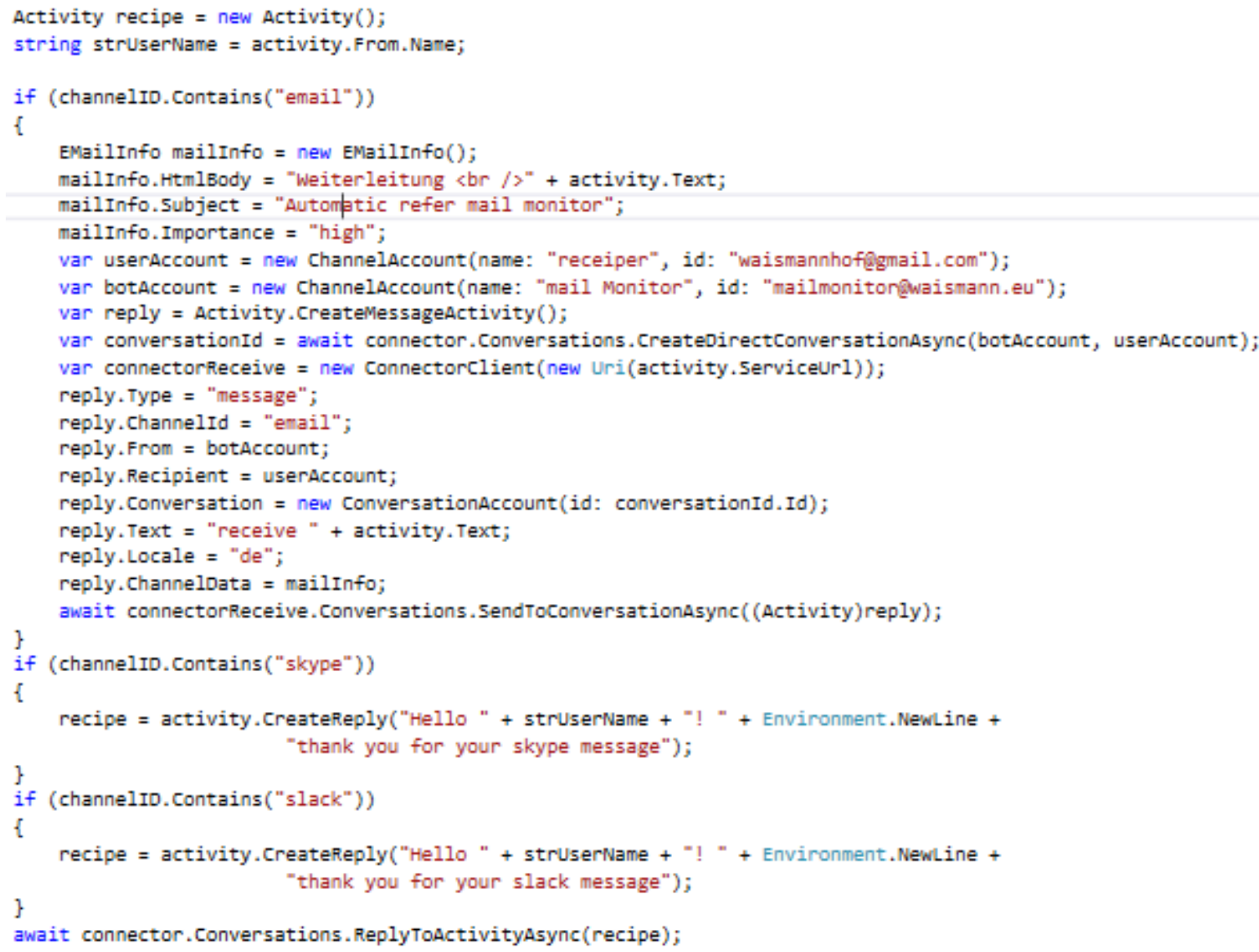

Figure 3: BotConnector with different channel handling

\subsection{Microsoft Bot Builder SDK for .NET}

The Bot Building SDK for .NET is a template, which can be used with the latest Visual Studio 2015 version. It provides a simple ASP.NET Web API including the MessageControll.cs. In this class, the message endpoint is defined and will be registered and hosted with the Bot Connector.

The template can be downloaded at GitHub and easily integrated and configured in Visual Studio or installed by Manage NuGet Packages. 
The Bot Framework provides powerful tools like an automatic translation to more than 30 languages and Cognitive Services like LUIS or the Microsoft Cognitive Services can be easily used by references.

For an easy start the template includes a simple "Echo Bot". The Echo Bot repeats user's messages and includes the total number of characters of the message. In Figure 4 shows the Post-Method of this "Echo Bot" [Pol17].

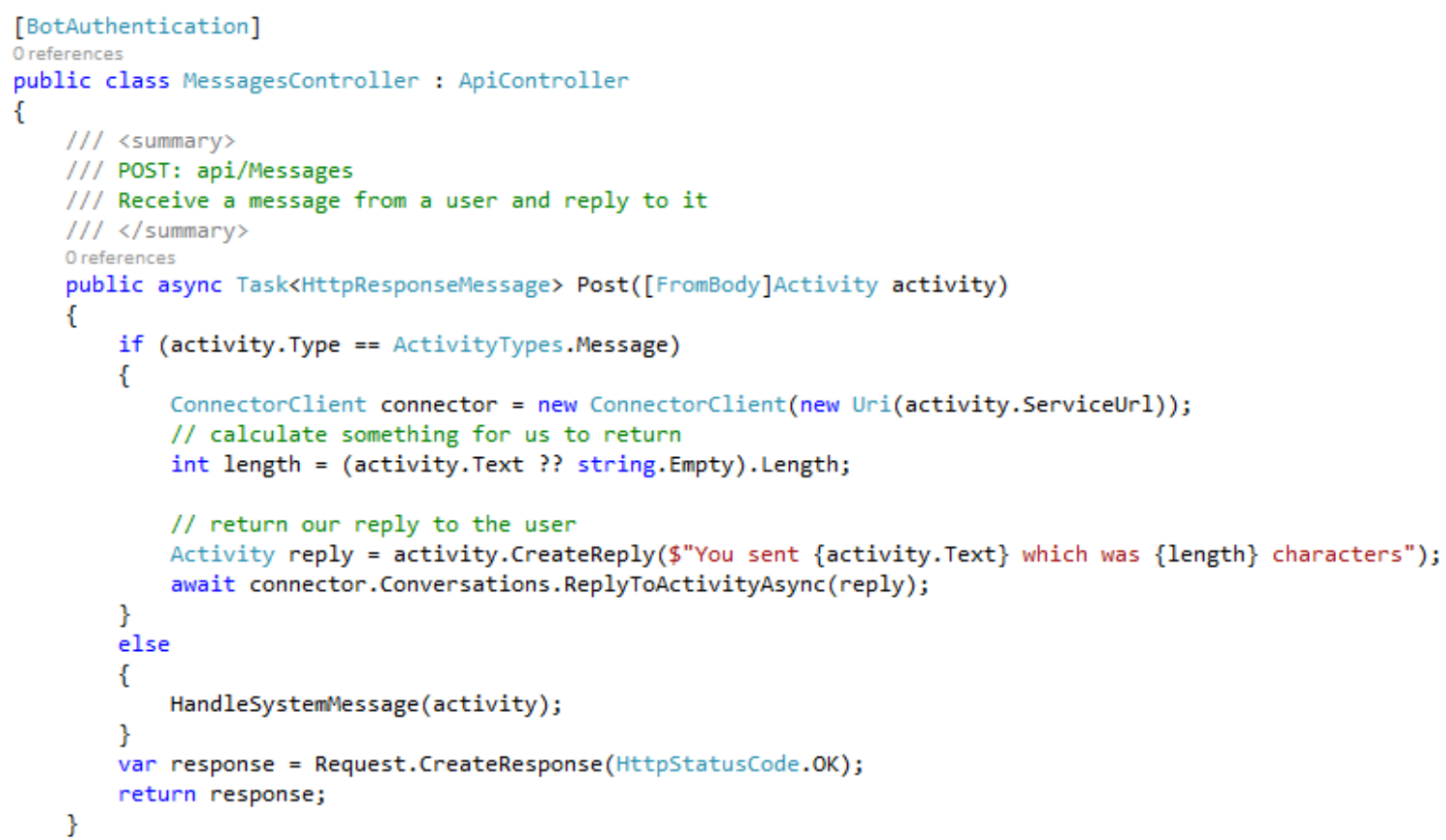

Figure 4: Simple "Echo Bot"-Post Method [Pol17]

For testing the bot Microsoft provides the Bot Framework Emulator which is a desktop application that allows testing and debugging the generated bots locally. The emulator displays the messages in a web chat UI and the logs of the JSON requests and responses.

This master thesis is about building an E-Mail monitoring \& management bot with Microsoft Social Bot. Therefore, the Microsoft Bot Framework with the communication channel Office365 Mail is used. 


\section{Selected Techniques from Artificial Intelligence}

\subsection{Overview}

One subdomain of artificial intelligence is data mining. It is simplified for Knowledge Discovery in Database (KDD) and it is a technique which extracts knowledge from lots of raw data. Data mining involves a variety of methods [FPS96]:

Different algorithms from the field of machine learning in the Artificial Intelligence can be roughly grouped in two main categories as:

\section{$\underline{\text { Supervised machine learning }}$}

The algorithm learns a function from given pairs of inputs (training data) and outputs (desired output value). During the learning, a teacher provides the correct function value for an input. The goal of supervised learning is to learn a general rule that maps inputs to outputs. Many learning algorithms such as [LeY09]:

- Classification: Assignment of objects to classes with common properties and accordingly prediction of attribute values.

- Regression: Identification of relationships between (multiple) dependent and independent variables.

\section{Unsupervised machine learning}

The algorithm generates a model for a given set of inputs that describes the inputs and allows for predictions. There are clustering methods that divide the data into several categories, which are distinguished by characteristic patterns. The network thus automatically creates classifiers by which it classifies the input patterns [LeY09].

- Clustering: Objects are classified into classes due to similarities (segmentation)

- Summarization: Reduction of the data set into more compact description without any significant information loss. 
Due to the various ways data mining can be done this master thesis will focus on classification which is described in more details in this chapter.

\subsection{Classification}

Classification attempts to find general structures in the existing data, so that conclusions can be drawn to new data, that is, a function of presentation is searched for a discrete value (class) or a numeric function value.

Definition: „Classification is the task of learning a target function $\mathrm{f}$ that maps each attribute set $\mathrm{x}$ to one of the predefined class labels $\mathrm{y}$. The target function is also known informally as classification model" [TSK05].

Methods of classification are Decision Trees, Naïve Bayes and Supported Vector Machines which are described in more details in this master thesis.

\subsubsection{Decision Trees}

Decision trees are one of the most popular approaches for representing classifier in data mining, machine learning and pattern recognitions. They are an ideal base learner for data mining applications because they are relatively fast to construct and produce interpretable models [HTF09].

\section{Basic concept}

A decision tree is constructed based on the "divide and conquer" [PAT12] paradigm and separates the data into several groups, determined by a rule with at least one condition. When learning from decision trees, the data are represented in an abstract tree and each branching represents a decision. Starting from the root, an attribute is queried at each branching and the next branching is selected. This procedure is continued until the end of the tree is reached. Since there are different possibilities for the trees, the algorithm tries to find the shortest and most optimal way. A decision tree pathway can be represented as an "if ... then ... else" structure. Learning from decision trees is one of the most frequently used methods in data mining. 
The complexity of a decision tree is explicitly defined by stop criteria and pruning methods and can be measured by using one of the following metrics:

- Number of internal nodes

- Number of leaf nodes

- Tree depth

- Number of attributes

The main components of a decision tree model are nodes and branches.

$\underline{\text { Nodes: }}$

A decision tree has three types of nodes:

- Root node: The root node is the first node of the tree and it has no incoming branch and two or more outgoing branches.

- Internal nodes: Each internal node represents a logical rule and it queries an attribute for deciding about the selection of the following node. An internal node has two or more outgoing branches.

- Leaf nodes: A leaf node represents the result of a combination of decisions and represents one of the classes respectively the class probabilities.

\section{Branches:}

Branches, also called edges, are the connection between two nodes and represents a decision from the nodes. "Each path from the root node through internal nodes to a leaf node represents a classification decision rule" [Son15].

The important steps in building a model are splitting, stopping and pruning.

Splitting: 
Beginning at the root a series of questions is asked which can all be answered with yes or no. For each node, a characteristic is queried and a decision is made. This is continued until all the features have been worked out and the leaves of the trees have been reached.

For this operation split-criterias are needed. They determine the rule for which the node of a decision tree splits. In most algorithms, the split functions are univariate and a node is defined only by the value of a node.

The nodes selection based on quality attributes such as:

- Entropy / Information Gain:

Entropy calculate the purity of any set of examples and the information gain is the expected reduction in entropy when a property is queried at a node. Information gain is based on Shannon's entropy and is used in ID3 and C4.5 as its attribute selection measure.

$$
\operatorname{Entropy}(S)=-\sum_{i=1}^{m} p(i) \log _{2} p(i)
$$

Entropy(S) is known as entropy of S [PAT12].

The Entropy is used to calculate the information gain.

$$
\operatorname{Gain}(S, A)=E(S)-\sum_{i} \frac{\left|S_{i}\right|}{|S|} * E\left(S_{i}\right)
$$

This simple split criterion provides good results but this still has a serious disadvantage, because it prefers attributes with many possible values. [QUI92].

- Gain Ratio:

The information gain uses attributes with many possible expressions. However, this can also be a disadvantage and can be remedied with a kind of normalization. Finally, after the determination of the information gain the result is divided by a normalization factor.

$$
\operatorname{Gain} \operatorname{Ratio}(A)=\frac{\operatorname{Gain}(A)}{\operatorname{SplitInfo}(A)}
$$


The SplitInfo(A) corresponds to the entropy of the attribute A.

$$
\operatorname{Split\operatorname {Info}}(A)=-\sum_{i=1}^{n} \frac{\left|T_{i}\right|}{|T|} * \log _{2}\left(\frac{\left|T_{i}\right|}{|T|}\right)
$$

The Gain Ratio represents the potential information generated when the training data amount $\mathrm{T}$ is divided into $\mathrm{n}$ sub-sets. Useful for the classification is the amount of information generated by the split.

\section{Stopping:}

To terminate the tree-growing process stopping conditions are to apply. The following examples are possible stop criteria:

- The training-set is empty, which means the tree closes with a leaf node. The decision tree generation is now terminated.

- All instances of the training set have the same value of the target attribute.

- The elements of the training set correspond to the stop criterion. The most likely target attribute expression is generated as a leaf.

- The maximum depth of the tree is reached.

- There is no longer an attribute to be examined.

When a stop criterion has been reached, the subtree is closed with a leaf node.

\section{Pruning:}

Induced learning can result in Overfitting in trees because the algorithm generates a classifier which perfectly fits the training data but has lost the capability of generalizing to instances not presented during training. In this case, the algorithm just memorizes the training instances instead of learning. In other words, if the stop criterion is not strict, the decision trees becomes very large and is over-adjusted in relation to the amount of training data. This over-adjustment can be corrected by Pruning. A Pruning method provides how to recognize the non-relevant subtrees. 
Two of the best-known Pruning methods are presented below.

- Pre-Pruning: In the Pre-Pruning, one tries to avoid all unnecessary nodes when creating the tree, since this procedure is however very complex, time consuming and partially inferior to the other method, it does not play any role in the subsequent view.

- Post-Pruning: First build the whole tree and then cut away unnecessary parts or form the tree around.

\section{Advantages [Mai10]:}

- Decision Trees are simple to understand, interpret, very simple and fast.

- They can handle nominal and numeric input attributes.

- Decision Trees are capable of handling dataset with possible errors or missing values.

\section{Disadvantages [Mai10]}

- The algorithms, ID3 or C4.5, require that the target attribute will have only discrete values.

- The "divide and conquer" method tend to perform well if a few highly relevant attributes exist, but less so if many complex interactions are present.

Algorithms like ID3 or C4.5 generate classifiers in form of decision trees [ROK08].

\subsubsection{ID3}

One of the basic algorithms for decision making is "Iterative Dichotomiser 3" (ID3) and was developed in 1986 by Ross Quinland. First, the algorithm searches for the most important property, which contains the highest Information Gain, for the root. This property represents the increase in the information content for the respective decision problem. In this way ID3 creates further nodes of the tree and thus, a decision tree is constructed from top-down (from the root to the leaves). The algorithm stops when all data series have the same value of the target attribute of the maximum Information Gain to be reached is less than 0 .

The ID3 algorithm uses a form of greedy search, thus, it picks the best attribute and performs no backtracking [LUG08]. 


\subsubsection{2. $\quad \mathbf{C} 4.5$}

C4.5 was developed as an extension of the ID3 algorithm by the same author Ross Quinlan. The C4.5 algorithm uses the Gain Ratio as a split criterion, thus eliminating the criticism of the ID3 algorithm. Another extension is the algorithm can handle numeric attributes and the errorbased pruning is performed after the growing phase [ROK08].

\subsubsection{Naive Bayes}

The Naïve Bayes classification is a statistical classification method that predicts the probability that an object belongs to a class. This classifier is based on the Bayes' Theorem. The Bayes classification is used to calculate the conditional probability of an event under a condition. Naïve Bayes classifiers simplify the problem by presupposing the validity of the independence assumption. That means that the effect of a characteristic on the classification is independent of other attribute values. This assumption is, in a sense, naive, since it is rarely valid but it significantly reduces the complexity of the problem.

\section{Basic concept}

In Naïve Bayes classifier, it is assumed that all the attributes of the records have a class or a function value with an independent probability (i.e. the probability with the Islamic belief, the technical course of studies, ... a terrorist). If this simplistic assumption were not made, the results would only be achieved by a considerable computational effort. The values for the independently assumed probabilities can then be calculated using the training data and the Bayes theorem.

The classification process has two basic procedures:

- Establish historical data set classification model,

- Use this model to sort the new data set [FUZ16].

Bayes theorem provides a way of calculating posterior probability $\mathrm{P}(\mathrm{c} \mid \mathrm{x})$ from $\mathrm{P}(\mathrm{c}), \mathrm{P}(\mathrm{x})$ and $\mathrm{P}(\mathrm{x} \mid \mathrm{c})$, as shown at the equation below. 


$$
P(c \mid x)=\frac{P(x \mid c) P(c)}{P(x)}
$$

- $\mathrm{P}(\mathrm{c})$ is the prior proba-bility of class.

- $\mathrm{P}(\mathrm{c} \mid \mathrm{x})$ is the posterior probability of class (target) given predictor (attribute).

- $\mathrm{P}(\mathrm{x} \mid \mathrm{c})$ is the likelihood which is the probability of predictor given class.

- $\mathrm{P}(\mathrm{x})$ is the prior probability of predictor.

Given the class variable $\mathrm{c}$ and a dependent feature vector $\mathrm{x}_{1}$ through $\mathrm{x}_{\mathrm{n}}$, Bayes' theorem states the following relationship:

$$
P\left(c \mid x_{1}, \ldots, x_{n}\right)=\frac{P\left(x_{1}, \ldots, x_{n} \mid c\right) P(c)}{P\left(x_{1}, \ldots, x_{n}\right)}
$$

Using the naïve independence assumption that

$$
P\left(x_{i} \mid c, x_{1}, \ldots, x_{i-1}, x_{i+1}, \ldots, x_{n}\right)=P\left(x_{i} \mid c\right),
$$

For all $i$, this relationship is simplified to

$$
P\left(c \mid x_{1}, \ldots, x_{n}\right)=\frac{P(c) \sum_{i=1}^{n} P\left(x_{i} \mid c\right)}{P\left(c \mid x_{1}, \ldots, x_{n}\right)}
$$

Since $\mathrm{P}\left(c \mid x_{1}, \ldots, x_{n}\right)$ is constant given the input, we can use the following classification rule:

$$
P\left(c \mid x_{1}, \ldots, x_{n}\right) \propto P(c) \sum_{i=1}^{n} P\left(x_{i} \mid c\right)
$$

Formula for Naïve Bayesian classification algorithm:

$$
\hat{c}=\arg \max _{c} P(c) \sum_{i=1}^{n} P\left(x_{i} \mid c\right)
$$

[SLD16]. 
Advantages of Naïve Bayes are [SBW99]:

- No several free parameters must be set that makes it to a very intuitive technique.

- The learning process did not require large amounts of training data.7

- It is computationally fast when making decisions.

\section{Disadvantages:}

- The Naïve Bayes classifier requires a very large number of records to obtain good results

- The class conditional independence, therefore loss of accuracy

\subsubsection{Support Vector Machine}

In 1995 Support Vector Machine (SVM) was developed for binary classification based on statistical learning theory by Vapnik. SVM is a supervised learning algorithm that analyse data and recognize patterns, used for classification and regression analysis.

\section{Basic concept}

SVM is performed to predict the output from a given input by mapping training data into vectors in some space. SVMs are hyperplanes that separate the training data by maximizing the margin. The vectors (data points) that lie closest to the hyperplane (or decision surface) are called support vectors and define the margin of largest separation between the two classes. The optimal hyperplane is in the middle of the margin as you can see in Figure 5. [COR95] 


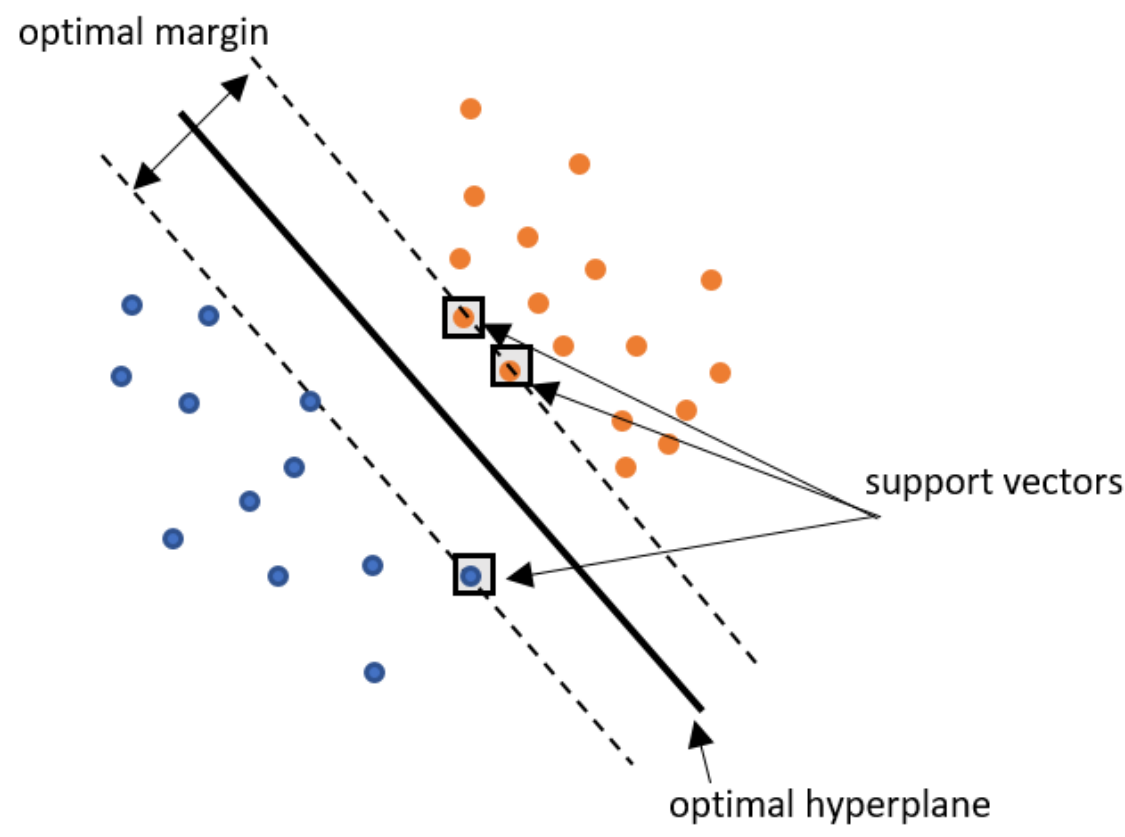

Figure 5: Simple linear support vector machine

Advantages [AuM08]:

- The produce of accurate and robust classification results.

- SVMs have less overfitting and are robust to noise.

Disadvantages [AuM08]:

- The lack of transparency of results.

- It is computationally expensive and it runs slow.

\subsection{Implemented Classification}

In this master thesis, the social bot is implemented with two different classification technologies, the decision tree with ID3 and Naïve Bayes.

The choice for the decision tree was made because decision tree based approaches learning from text are particularly appealing, since rules and trees provide explanatory insight to 
developers. The accurate and interpretable models with relatively little user intervention can be built very fast and can also be efficiently parallelized.

The Naïve Bayes algorithms was chosen because it makes use of the variables contained in the data sample, by observing them individually, independent of each other.

SVMs are another promising candidate for classification, but the objective of this thesis was to demonstrate in general how to integrate AI classification in general into the MS Bot Framework, thus a comparison of two different techniques was considered to be sufficient.

Both algorithms are integrated into the Bot-application with the Microsoft Accord.Net Framework and in chapter 7, the evaluation of these two classifications is shown.

\section{Implementation Details}

This chapter describes the implementation of the social bot with artificial intelligence. The social bot is a web service based on the Microsoft BotFramework and is connected with the Email Channel. For the decision making with artificial intelligence two technologies of classification, decision tree ID3 and Naïve Bayes, are chosen.

At first, the process sequence is briefly described and subsequent all implemented technologies are specified.

\subsection{Data Flow Process}

The data flow chart is shown in Figure 6. 


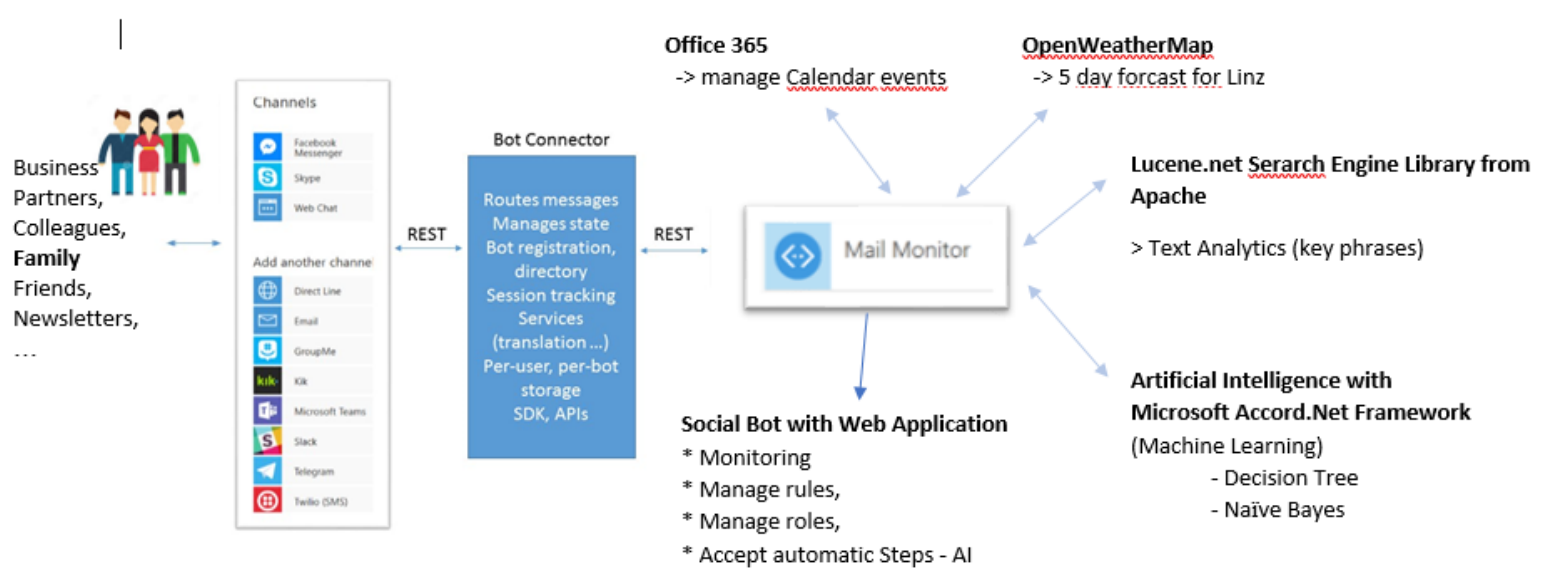

Figure 6: Mail Monitoring Bot - Flow Chart

After the bot receives an incoming email the bot searches in his contacts for the sender's role identification, for the decision the role id is needed. After that the bot starts with the text analytics.

First a string object with email subject and email body is generated and send to the Lucene.Net for text analytics. Lucene.Net is an open source search engine library and this service sends key phrases of the text back to the bot.

Next step is the verification whether the text is an appointment request. Therefore, the bot verifies the key phrases for specified keywords. Specified keywords for an appointment request are: appointment, time to come, have time. The simple method CheckOfAppoinment and the keywords are shown in Figure 7. 


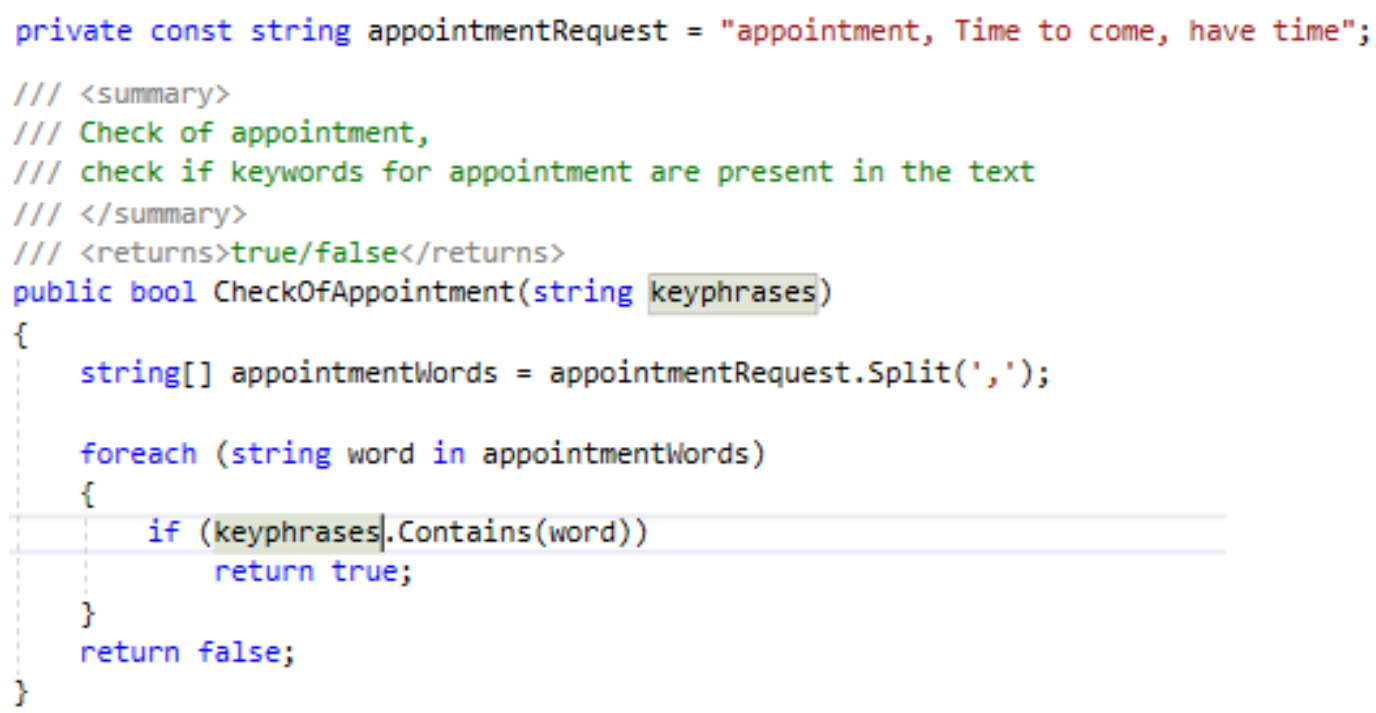

Figure 7: CheckOfAppoinment method

If the verification has been successful the next step is to find out on witch date and time the appointment will be held. In this verification date and time data where analysed and stored in a DateTime object. Two rules were defined therefore:

- Keywords like today and tomorrow with exact time data are converted in a DateTime.Today and DateTime.Today.AddDays(1) object, adding the exact time data to the object.

- The keyword date must be before an exact date data. Eg. date 07. 072017 13:00.

For the exact time data, the bot can differ between the 12-hour and 24-hour format.

If the date for the appointment is in the future the bot continued with the type analysis. For the classification, it is important to find out of which type the appointment is. The bot differs between four types with special keywords:

- Outdoor sport: skiing, hiking, swimming

- Indoor sport: tennis, climbing, workout, gym

- Wellness: wellness, sauna,

- Entertainment: cinema, café, eat, lunch, dinner, tea

- Default: everything else 
For the decision, it is necessary to know how the weather will be on this date. Therefore, the bot calls the online service OpenWeatherMap for a 5-day forecast and stores the weather information for the decision.

The last information the bot is still needed for the decision is the information if there is still a free date in the calendar available at this time. Microsoft offers the Office 365 API to provide access to customer's Office 365 data. On this process time, the bot calls all event information in the calendar for this date with the Office 365 API. The information Yes is stored for free appointment and No for not available appointment for the decision-making process.

For the decision-making process two different classifications are implemented, the decision tree with ID3 and Naïve Bayes. After this process, an answer mail is send to the sender.

\subsection{Microsoft Bot Application}

This master thesis is based on the previous practicum project of Manuela Pollak, Social Bots with Microsoft Bot Framework [Pol17]. In this paper, the implementation of Microsoft Bot Application is described in detail and the resulting Mail Monitoring Bot was taken over for this master thesis. Therefore, only the most important details of installation and configuration of Microsoft Bot Application will be briefly described here.

To deploy the social bot with Microsoft Bot Framework those steps have to be taken:

- Install the latest version of Visual Studio 2017.

- Update all Visual Studio extensions.

- Install the Bot Application template for .Net.

- Publish the bot to Azure.

- Register the bot on the Developer Portal.

- Get an Office 365 Account with an Exchange Server.

- Register the bot with this Office 365 Email address to the Email Channel.

Figure 8 shows the Post method from the MessangerConroller. This method receives the user's messages from any possible Channels. The delivered Activity object contains the Channel 
information in the object ChannelId. If the message comes from the Email-Channel the Activity object will be delivered to a background thread worker for analysing the text message.

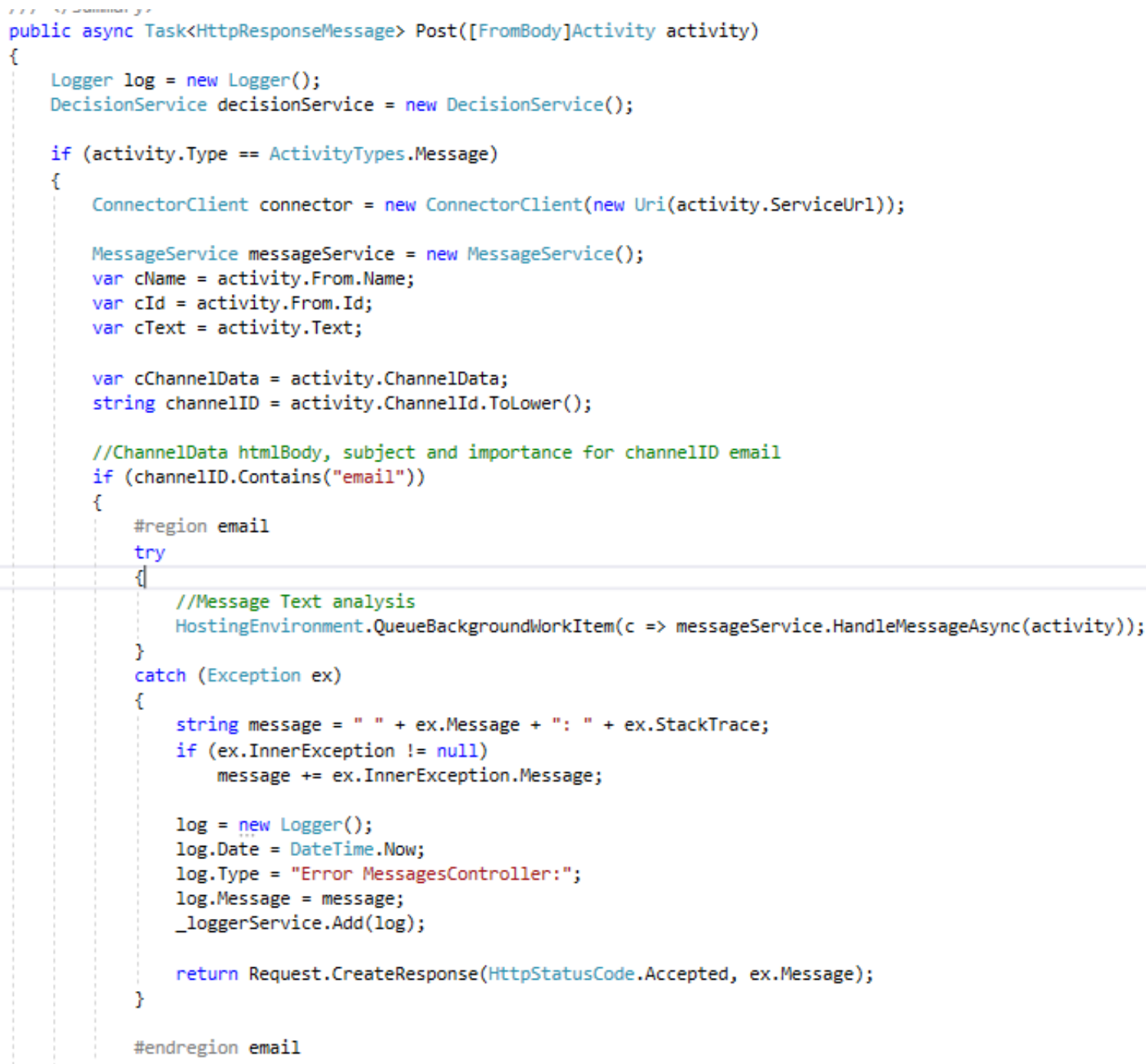

Figure 8: Bot Framework - Post method 


\subsection{Entity Framework, Repository, Unit of Work Pattern and Microsoft SQL Database}

This chapter is about storing data in database and how it is integrated in the social bot application. This and the database tables will be described briefly.

Entity Framework Code First is used for defining models in Visual Studio and creating the database automatically on the fly. The bot application uses the Microsoft Azure SQL Database and all database operations are defined in a generic repository class and Unit of Work is referred to as a single transaction that involves multiple operations. In other words, the repository and unit of work patterns create an abstraction layer between the data access layer and the business logic layer [DYK13], as it can be seen in Figure 9.

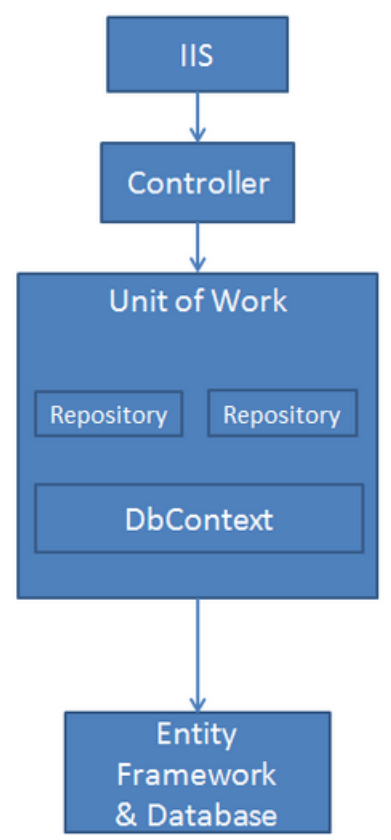

Figure 9: EF, Unit of Work, Repository, Database [DYK13]

Figure 10 shows the Microsoft SQL Class diagram, this classes/tables are used from the bot application:

- Decisions: In this table, all training data for the classification are stored.

- Emails: All incoming emails with the text analytic results are stored in this table.

- Loggers: In this table, all log information and intermediate steps and results are stored from the bot application. 
- Settings: This table is about the settings for the bot and for the website. On this time only the information about which decision type is to use is stored here.

- People: All sender from incoming emails are stored in this table. The most important information of a sender is his role in the decision-making process.

- Roles: In this table, all possible roles of senders are stored.

- ClassificationResults: In addition to the decision-making information, all results of the decision-making process are stored in this table.
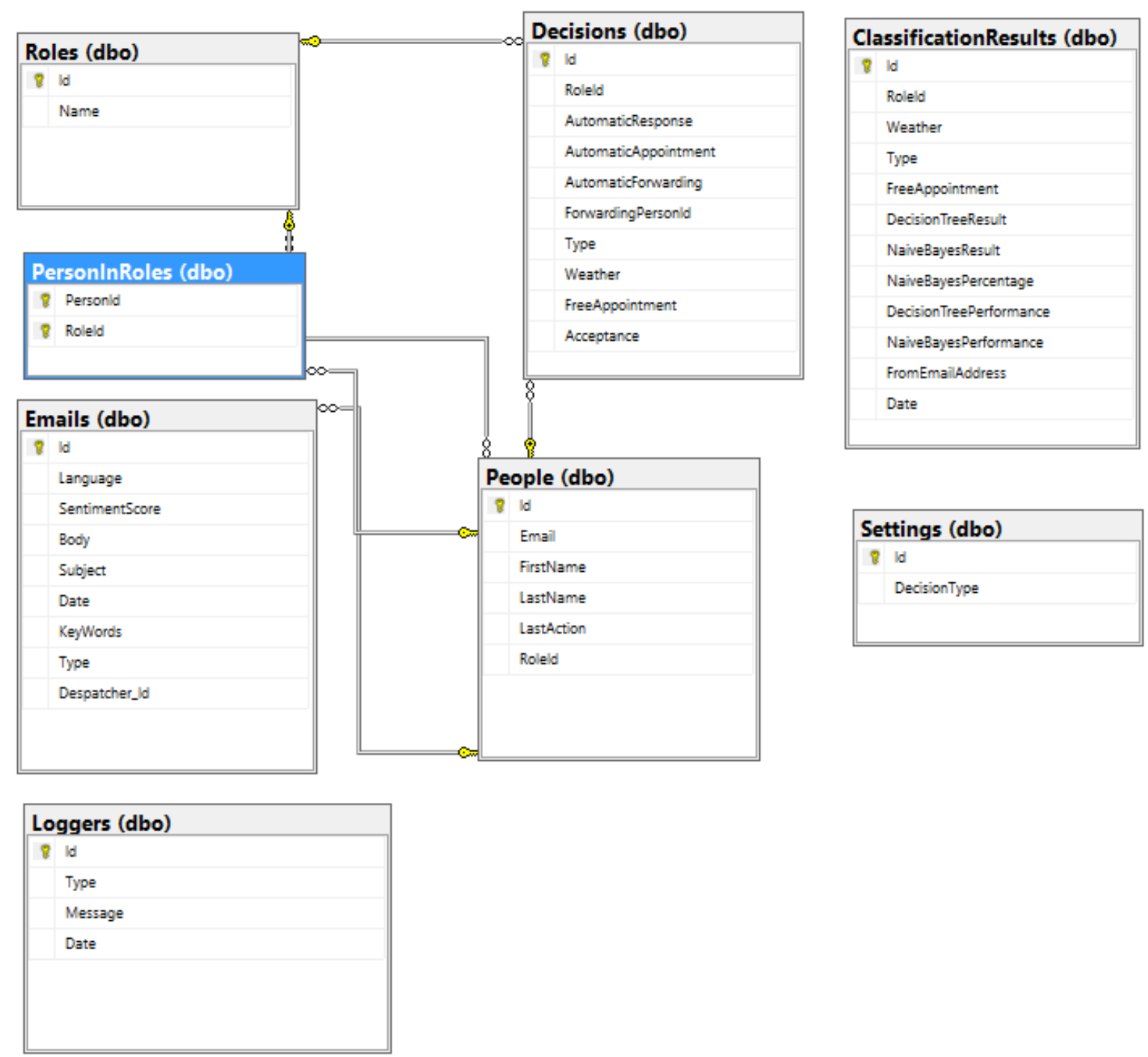

Figure 10: MS SQL - Class Diagram 


\subsection{Preliminary work classification}

The input data for a classification task is a collection of records. Figure 11 shows the sample training dataset used for classifying appointment request acceptance into true or false.

The decision affected of the attribute set includes properties of the appointment:

- RoleId, this represents the Role of sender, there are two possibilities:
○ 1 for Role: Family
○ 2 for Role Business

- Free appointment: is a free appointment available in the calendar, two possibilities:
○ Yes
○ No

- Type: six different types are possible:
○ Indoor Sport,
○ Outdoor Sport,
○ Wellness,
○ Entertainment,
○ Business,
○ Default

- Weather. nine different weather types are possible:
○ broken clouds,
○ clear sky,
○ few clouds,
$\circ$ light rain,
o rain,
○ scattered clouds,
○ whatever
- moderate rain
$\circ$ heavy intensity rain

- Acceptance: is the appointment request accepted, two possibilities:

- Yes 
○ No

\section{Decisions - Training data}

Create New

\begin{tabular}{|c|c|c|c|c|}
\hline Roleld & FreeAppointment & Type & Weather & Acceptance \\
\hline 1 & 口 & Wellness & few clouds & $\square$ \\
\hline 1 & 四 & Wellness & clear sky & $\square$ \\
\hline 1 & $\square$ & Wellness & rain & $\square$ \\
\hline 1 & $\square$ & Wellness & moderate rain & $\square$ \\
\hline 1 & $\square$ & Outdoor Sport & light rain & $\square$ \\
\hline 1 & 口 & Outdoor Sport & rain & $\square$ \\
\hline 1 & 四 & Outdoor Sport & few clouds & 口 \\
\hline 1 & $\square$ & Outdoor Sport & broken clouds & $\square$ \\
\hline 1 & 口 & Outdoor Sport & whatever & $\square$ \\
\hline 1 & 口 & Outdoor Sport & clear sky & $\square$ \\
\hline 1 & 四 & Outdoor Sport & heavy intensity rain & $\square$ \\
\hline 1 & 田 & Outdoor Sport & scattered clouds & 口 \\
\hline 1 & 口 & Indoor Sport & clear sky & $\square$ \\
\hline 1 & 田 & Indoor Sport & light rain & 口 \\
\hline 1 & $\square$ & Indoor Sport & whatever & $\square$ \\
\hline 1 & 田 & Indoor Sport & broken clouds & $\square$ \\
\hline 1 & 口 & Indoor Sport & heavy intensity rain & 四 \\
\hline 1 & 四 & Indoor Sport & rain & 曰 \\
\hline 1 & 口 & Indoor Sport & few clouds & 曰 \\
\hline 1 & 口 & Entertainment & clear sky & $\square$ \\
\hline 1 & $\square$ & Entertainment & scattered clouds & $\square$ \\
\hline 1 & $\square$ & Entertainment & few clouds & $\square$ \\
\hline 1 & 口 & Entertainment & moderate rain & $\square$ \\
\hline 1 & 四 & Entertainment & heavy intensity rain & 口 \\
\hline 1 & $\square$ & Default & whatever & $\square$ \\
\hline 1 & 四 & Default & clear sky & $\square$ \\
\hline 1 & $\square$ & Default & light rain & $\square$ \\
\hline 1 & 口 & Default & few clouds & $\square$ \\
\hline 1 & 四 & Default & heavy intensity rain & 田 \\
\hline 2 & $\square$ & Business & clear sky & 口 \\
\hline
\end{tabular}

Figure 11: Decisions training data 
As training data, 26 types were selected with different feature combinations, for which it was known whether a term should occur. Based on these training data, the Decision Tree depicted has been determined, see Figure 12.

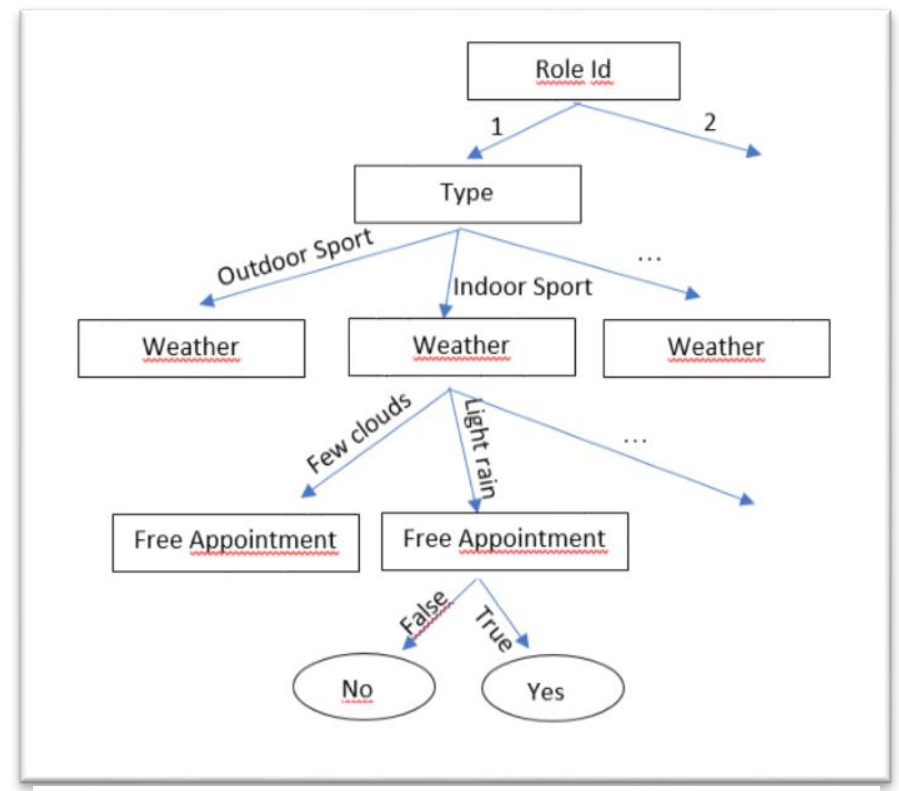

Figure 12: Decision Tree from training data

In the future, e.g. a new appointment request with these attributes:

- $\quad$ RoleId $=1$,

- $\quad$ Type = Indoor Sport,

- Weather = light rain,

- $\quad$ Free appointment $=$ true

can be classified as an appointment which will always be accepted with the Decision Tree.

This example is something different with Naïve Bayes. Because of the calculation of probability, the decision doesn't accept the appointment request. More about the different result of this and other examples are shown in chapter 7, Evaluation. 


\subsection{Apache Lucene.Net}

Lucene.Net is a part of the Lucene search engine library ${ }^{17}$ from the Apache Software Foundation. This open source full-featured text search engine is for the .NET runtime and written in C\# language. The latest release is available via NuGet. Lucene.Net can be easy included in the Visual Studio Project via the NuGet package manager.

The social bot uses Lucene.Net for the email body text analysis and tokenizing. Therefore, the StandardAnalyzer Class is implemented. The StandardAnalyzer filters StandardTokenizer with StandardFilter, LowerCaseFilter and StopFilter, using a list of English stop words. This Analyzer includes the TokenStream Class. With this class, all relevant attributes are retrieved from the email body text to be analyzed. All single attributes are saved in the string object called keywords. Figure 13 shows the Method GetKeywords. The keywords are stored in a String object separated with semicolon.

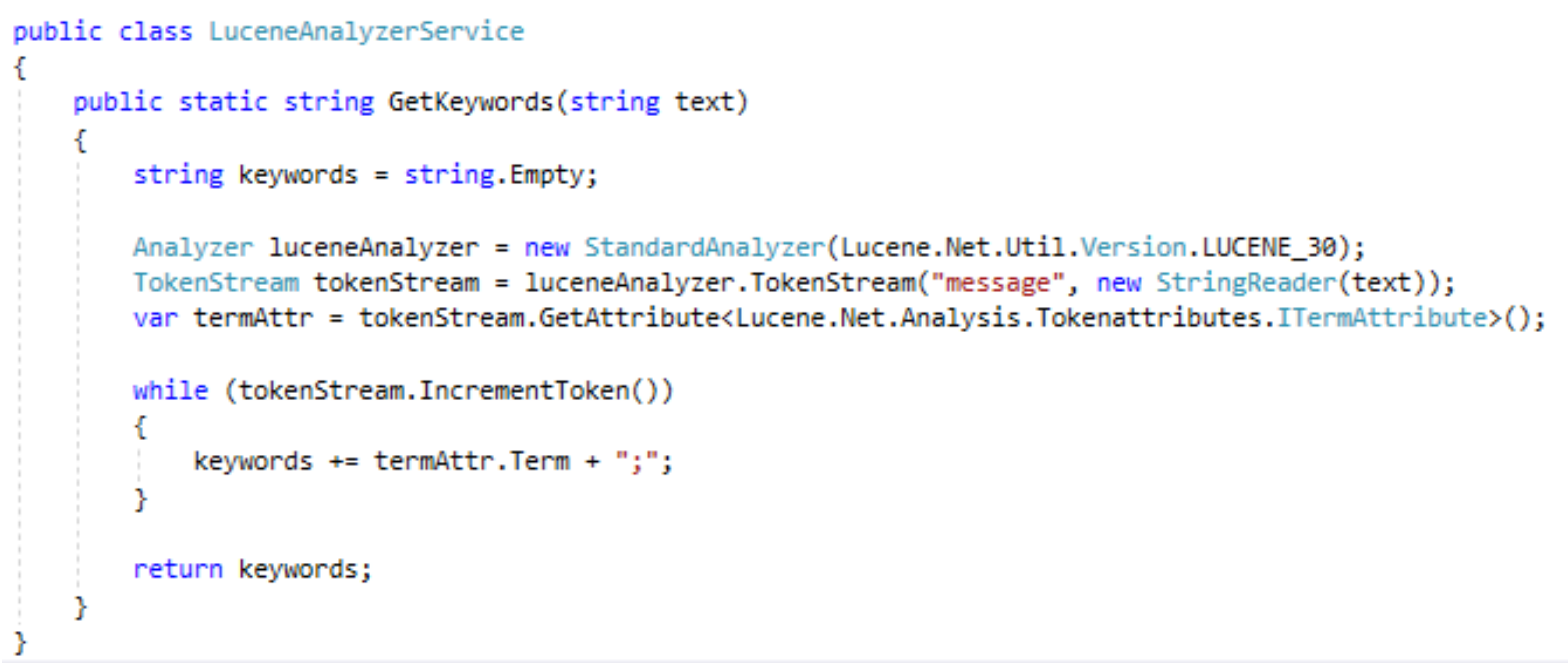

Figure 13: LuceneAnalyser GetKeywords Method

Figure 14 shows an incoming email body text which the social bot delivers to the GetKeywordsmethod in the LuceneAnalyzerService.

\footnotetext{
${ }^{17} \mathrm{https}: / /$ lucenenet.apache.org
} 
Subject: Appointment

Dear Manuela,

we are going to the mountain for hiking on date 09. 072017 07:30

are you coming with us?

CU

Manu

Figure 14: Incoming Email Body

The returned keywords from the email body text of Figure 14 are shown in Figure 15.

dear;manuela;we;going;mountain;hiking;date;09;07;2017;07;30;you;coming;us;cu;manu; appointment;

Figure 15: Lucene.Net Keywords

\subsection{OpenWeatherMap}

For the decision-making process, it is necessary to know about the weather on the request date. Therefore, the online service OpenWeatherMap (OWM) is implemented. OWM appears to offer the simplest and most extensive features. The service provides an API for weather information to developers with HTML, JSON and XML endpoints.

Using OWM requires an API key. After signup for the free account on the website a key will be created and can be located in the profile page. This key must be used for any request made to the weather service. The free account includes amongst others the current weather data and 
a five-day forecast and allows 60 calls per minute. The five-day forecast includes weather data every three hours.

The request is implemented in a separate class in the folder Services. The social bot application calls the API via WebClient. The condition URL has this format:

http://api.openweathermap.org/data/2.5/forecast?q=Linz\&appid=key\&units=metric\&mode=xml

These parameters are set to the request URL:

- $\mathrm{q}$ : is the location of interest. The bot application has set Linz in a constant string object Linz.

- mode: indicates the returned result format, in this application the XML format is always set.

- appid: this is the developer key, located in the profile page on the website.

- units: for temperature in Celsius metric is used.

In Figure 16 the Code of the WeatherService with the GetWeather method is shown and in Figure 18 a short cut of a five-day XML call for Linz is shown.

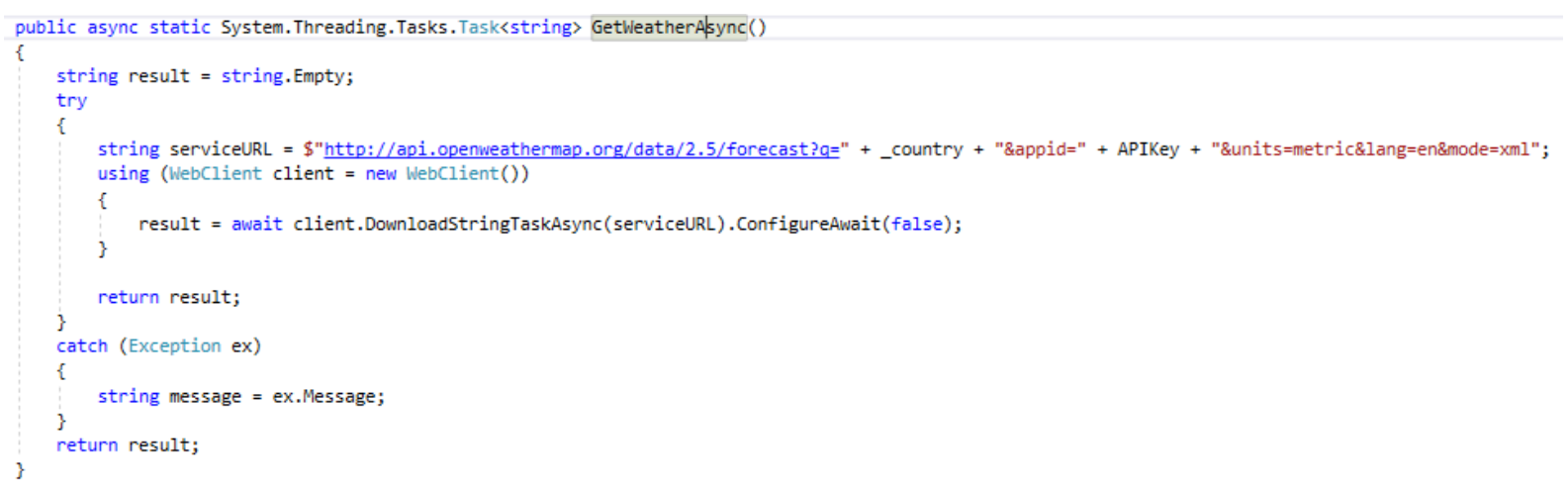


Initially the result XML data is unformatted stored in a string object. For better reading the model class Weatherdata was created with the OWM XML-structure and with the XmlSerializer Class the string object can be deserialized and modelled to the weather model class, shown in Figure 17.

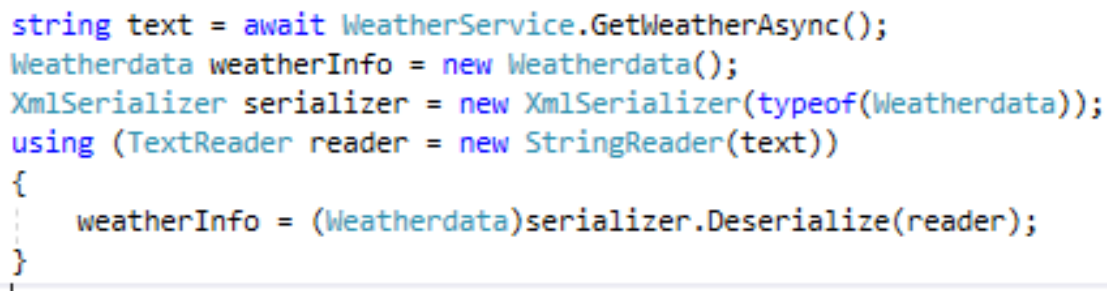

Figure 17: Deserialize XML string object to weather model class

If the appointment request date is not between the five-day forecast the information whatever is stored, in the column Weather, for the decision.

These forecast data are necessary for the social bot:

- Time To: This date is compared with the given date in the email body.

- Symbol Name: This symbol name is set to the decision-object as weather information. 


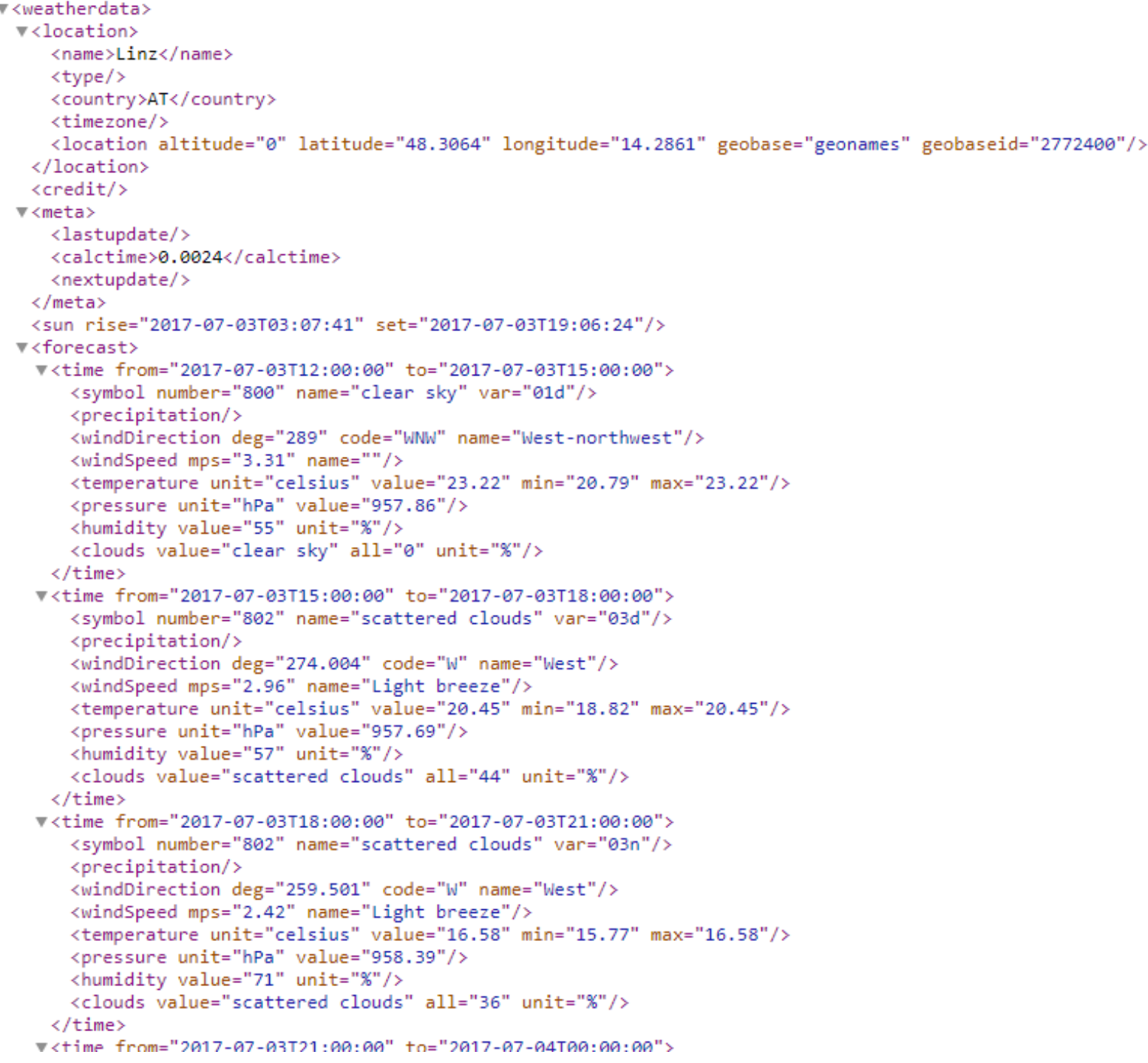

Figure 18: Example OpenWeatherMap API Call

\subsection{Accord.NET Framework}

The Accord.NET Framework is a C\# framework designed for developers in the fields of Artificial Intelligence including the topics of machine learning, computer vision and even more.

For the decision-making process, the Decision Tree and Naïve Bayes was implemented. 


\subsubsection{Decision Tree}

There are two decision tree learning algorithm possibilities called ID3 and C4.5. For the social bot only the decision tree with ID3 was implemented.

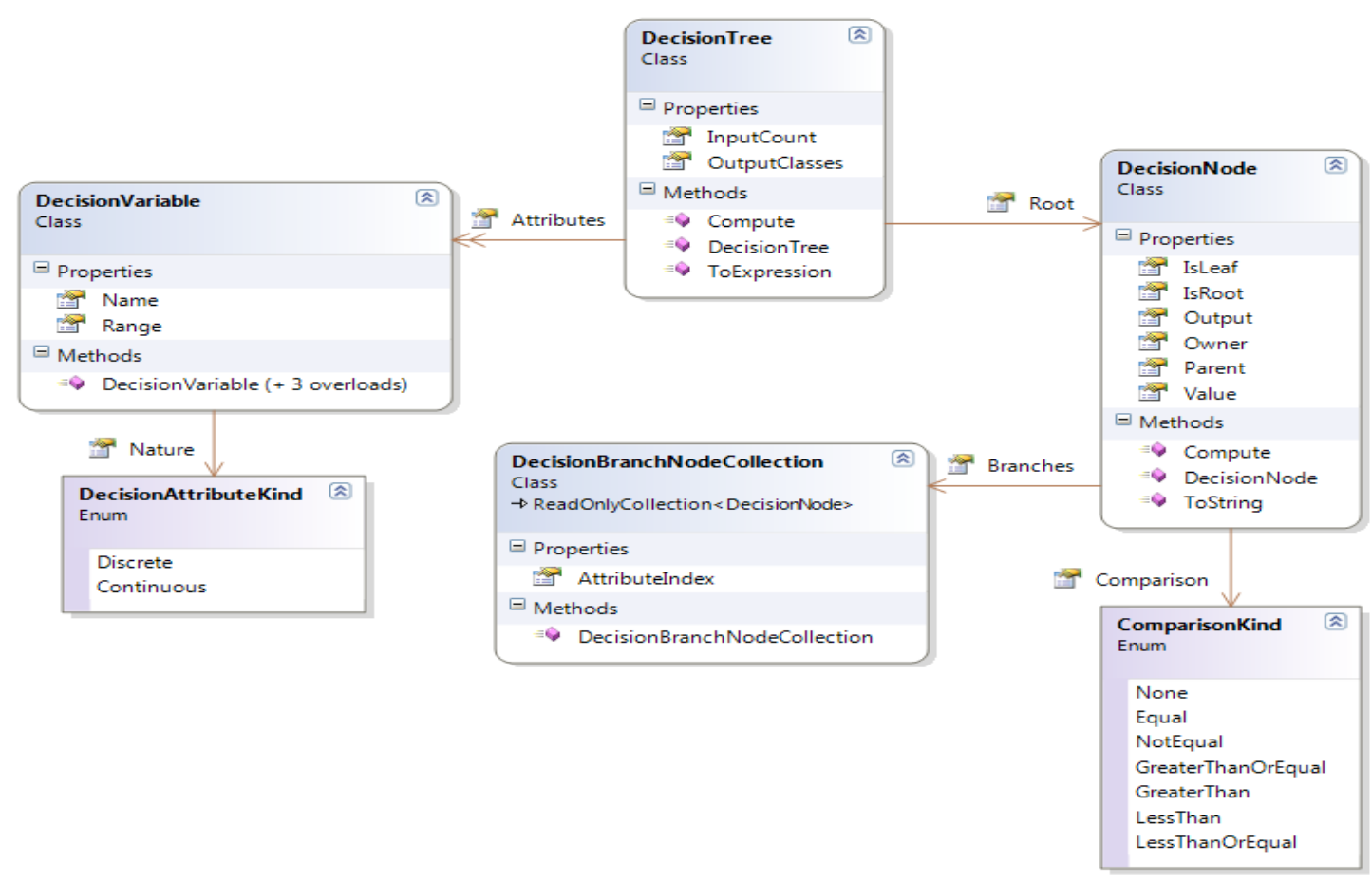

Figure 19: Accord.MachineLearning.DecisionTree namespace [Sou12]

The decision tree namespace called Accord.MacineLearning.DecisionTree. Figure 19 shows the class diagram with those relationships. Following them the classes are described briefly [Sou12] with some implementation details:

- Decision Tree: this is the main class and represents the decision tree. To compute the classification from an input vector the method Compute is given. In the newest framework version, the method Compute is obsolete and method Decide is used instead as you can see in figure 16 .

- Decision Node: this is the node class. A decision tree may have children nodes but it may not have.

- Decision Variable: in this class, the nature of each variable is specified. In Figure 17 the implementation in the social bot can be seen. 
- DecisionBranchNodeCollection: this class is a collection of children nodes containing with information about which attribute of the data should be compared with the child node.

\section{Implementation:}

The decision tree implementation uses a codification to convert string variables into discrete symbols. First the input data which are get from a database are represent in a DataTable. After that the input data (training data) are converted by the Codification into integer symbols, shown in Figure 20.

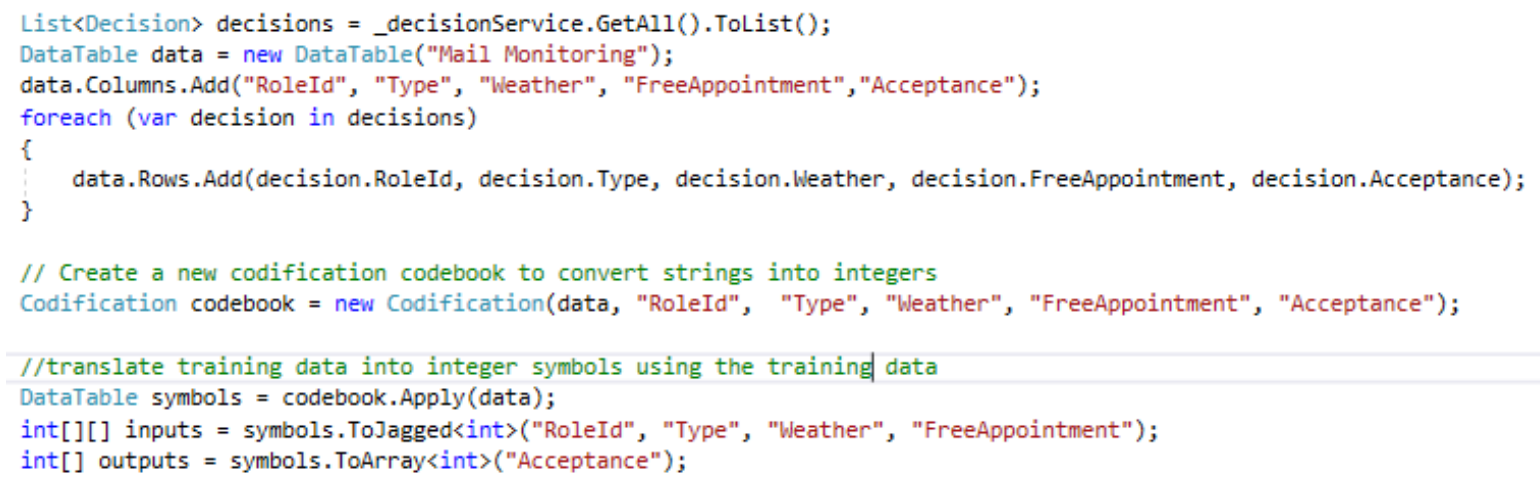

Figure 20: Implementation Decision Tree, Codification

Next step is the declaration of the DecisionVariable with the declaration of possible values. After creating the main class DecisionTree with their variables the ID3 learning algorithm is created and ready to learn the training instances, shown in Figure 22.

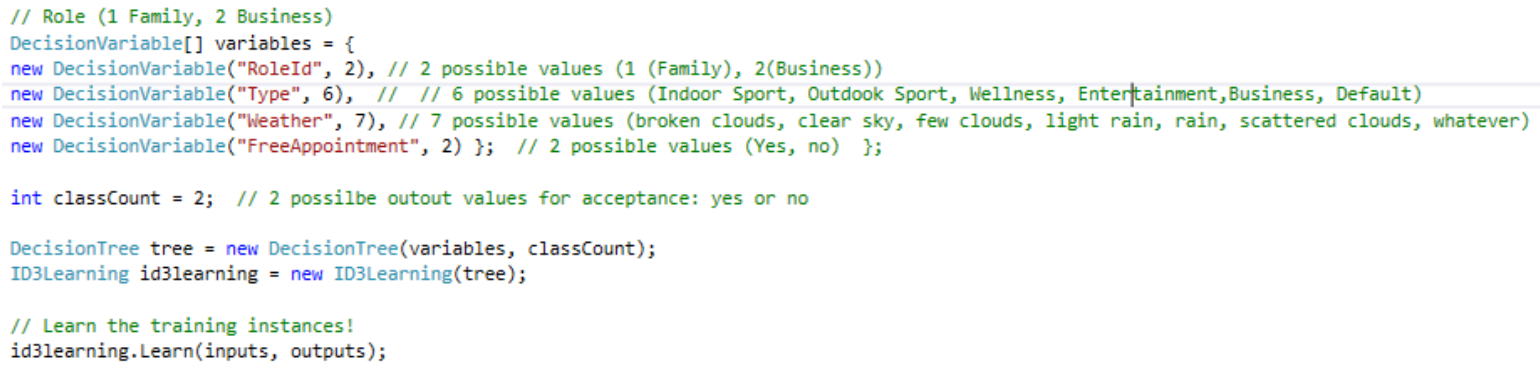

Figure 22: DecisionVariable from social bot 
After that, the tree can be queried for the information of the appointment request through its Decision method. Method Revert converts the return value of Decision into a string answer, yes or no. This is shown in Figure 23.

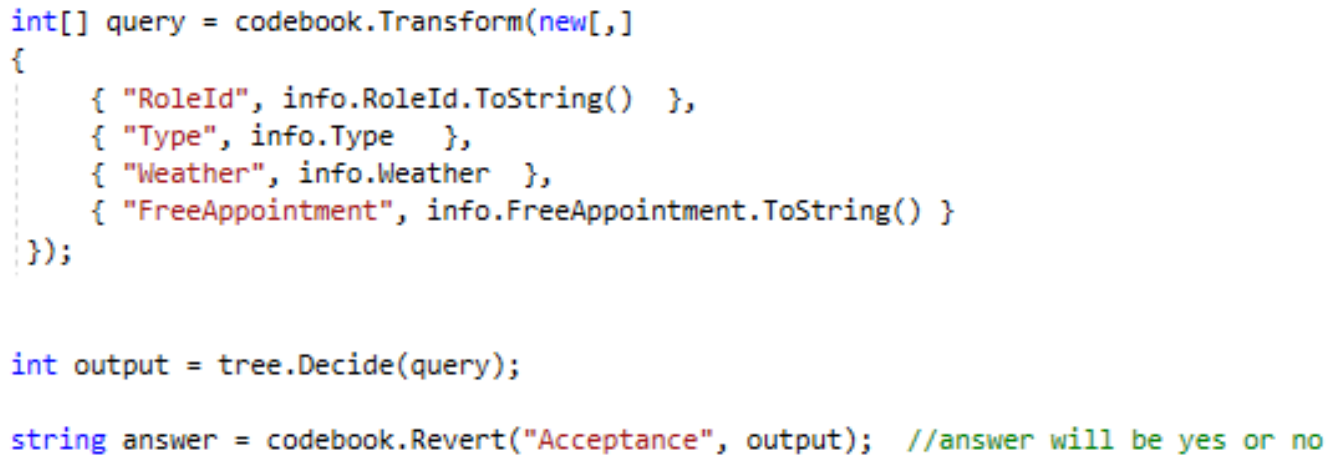

Figure 23: Decision Tree Transform and Decision

\section{New decision rule entry}

If the input data are too different to the training data than the Decision Tree implementation recognise this by an exception and a new decision object with the input data will be created. This happens during the test phase with this input data:

- Role Id: 1

- Type: Entertainment

- Weather: moderate rain

- FreeAppointment: true

Therefore, a new row in the decision table was generated, as it can be seen in Figure 24. On the website, which provides a user interface for monitoring and controlling the algorithm behaviour, there is the possibility to change individual values or to create new decision rules manually.

\begin{tabular}{|c|c|c|c|c|c|c|c|c|}
\hline Roleld & AutomaticRes... & AutomaticApp... & AutomaticFor... & ForwardingPer... & Type & Weather & FreeAppointm... & Acceptance \\
\hline 1 & False & True & False & 4 & Entertainment & moderate rain & True & True \\
\hline 1 & Ealm & Trin & Ealm & $A$ & Indaner Sunart & hreatean -lavidr & Trun & Trun \\
\hline
\end{tabular}




\subsubsection{Naive Bayes}

Accord.Net's Naive Bayes classifier is a simple probabilistic classifier based on Bayes' theorem with strong independence assumptions between the features [ACC17].

\section{Implementation:}

First the social bot gets all training data from the database and stores them into a DataTable with columns for "RoleId", "Type", "Weather", "FreeAppointment" and "Acceptance”. Naïve Bayes can be trained directly with string objects but number symbols are more easily representable so they will convert to use of a codebook. The codebook transforms all values into an integer symbol. For example, the values of column "Weather" could be represented like this:

- “Indoor Sport” by “0”

- "Outdoor Sport" by "1"

- "Wellness" by " 2 "

- "Entertainment" by “3”

- "Business" by "4"

- "Default" by "5"

After the codebook is generated the input and output pairs are extracted to train. This process is shown in Figure 25.

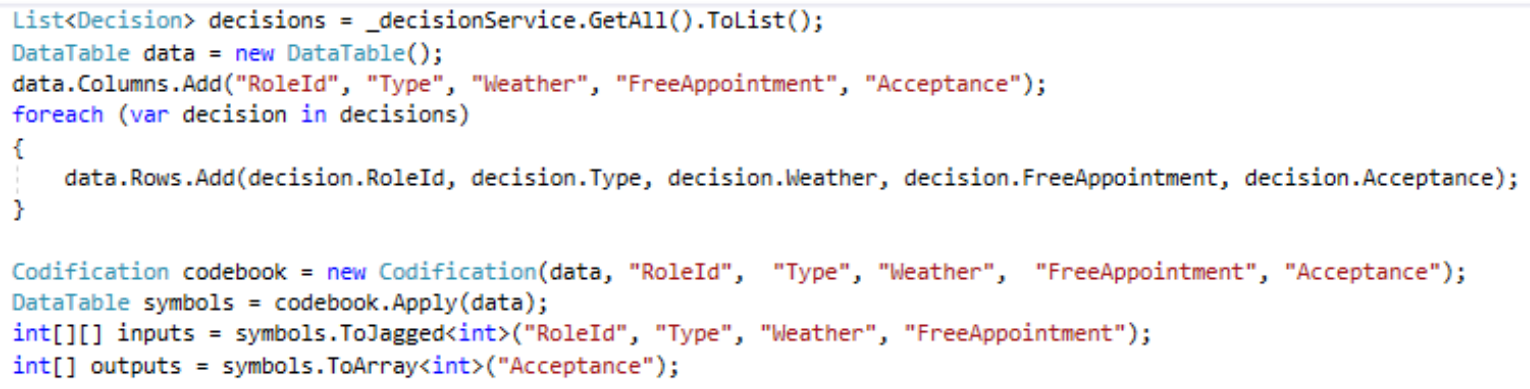

Figure 25: Naive Bayes Implementation 1

In Figure 26 the next steps are shown. The NaiveBayesLearning model is created and the method Learn will learn from the training data. A model to predict the last resulting column 
"Acceptance" is built. The others column "RoleId", "Type", "Weather" and "FreeAppointment" will be used as predictors. A predictor is variable which is used for the decision.

After that, the social bot queries the classifier for new appointment request information through the Decide method. This method obtains a numeric output that represents the answer, " 0 " for "No" and "1" for "Yes". With the Revert method this numeric output can be converted to an actual "Yes" or "No" answer. The Naïve Bayes class also allows the developer to extract the probabilities for each possible answer with the method Probabilities.

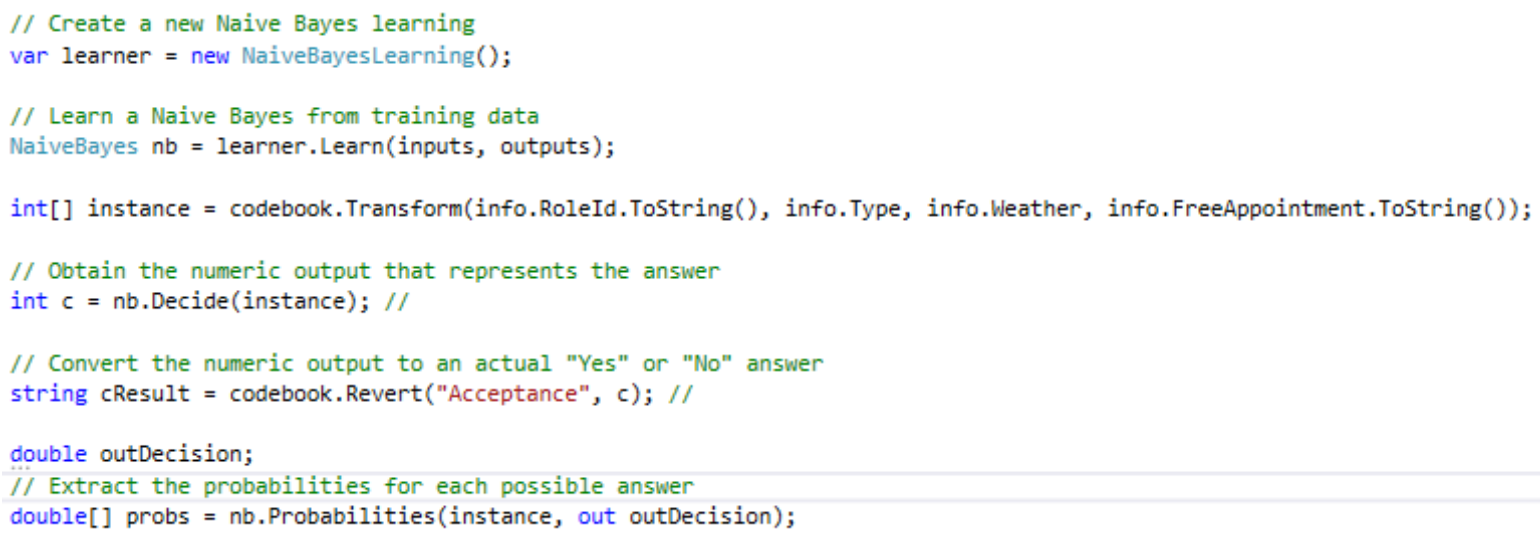

Figure 26: Naive Bayes Implementation 2

\subsection{Office 365 API}

The Office 365 API enables the social bot application to provide access to its Office 365 data and it is used on several times:

- Classification: Check for free appointment on a several date and time.

- Classification: Set a new appointment to the calendar.

- Management and Monitoring Website: view incoming Emails

- Management and Monitoring: CRUD (create, read, update and delete) Calendar Events.

For the successful use of Office 365 API more several steps are needed:

- Registration on Active Directory on Azure

- Office 365 Developer Portal, to get a Tenant ID 
- Install the Azure Directory Authentication Library (ADAL)

- Install OWIN packages

- Install Microsoft Office 365 Outlook Service

The bot application includes a single-tenant MVC that uses Azure Active Directory (AD) Single Sign-on to authenticate. Therefore, a persistent Active Directory Authentication Library (ADAL) token cache that uses a local database for caching is employed. ADAL enables the bot to authenticate to Active Directory and obtain access token for security API calls. In addition to ADAL, OWIN ${ }^{18}$ is also used for the authentication. OWIN is a standard interface between .NET web servers and web applications for decouple server and application.

For this it is necessary that the bot application is configured to use Secure Socket Layers (SSL) and to set the bot appropriate redirect URL into the Office 365 services.

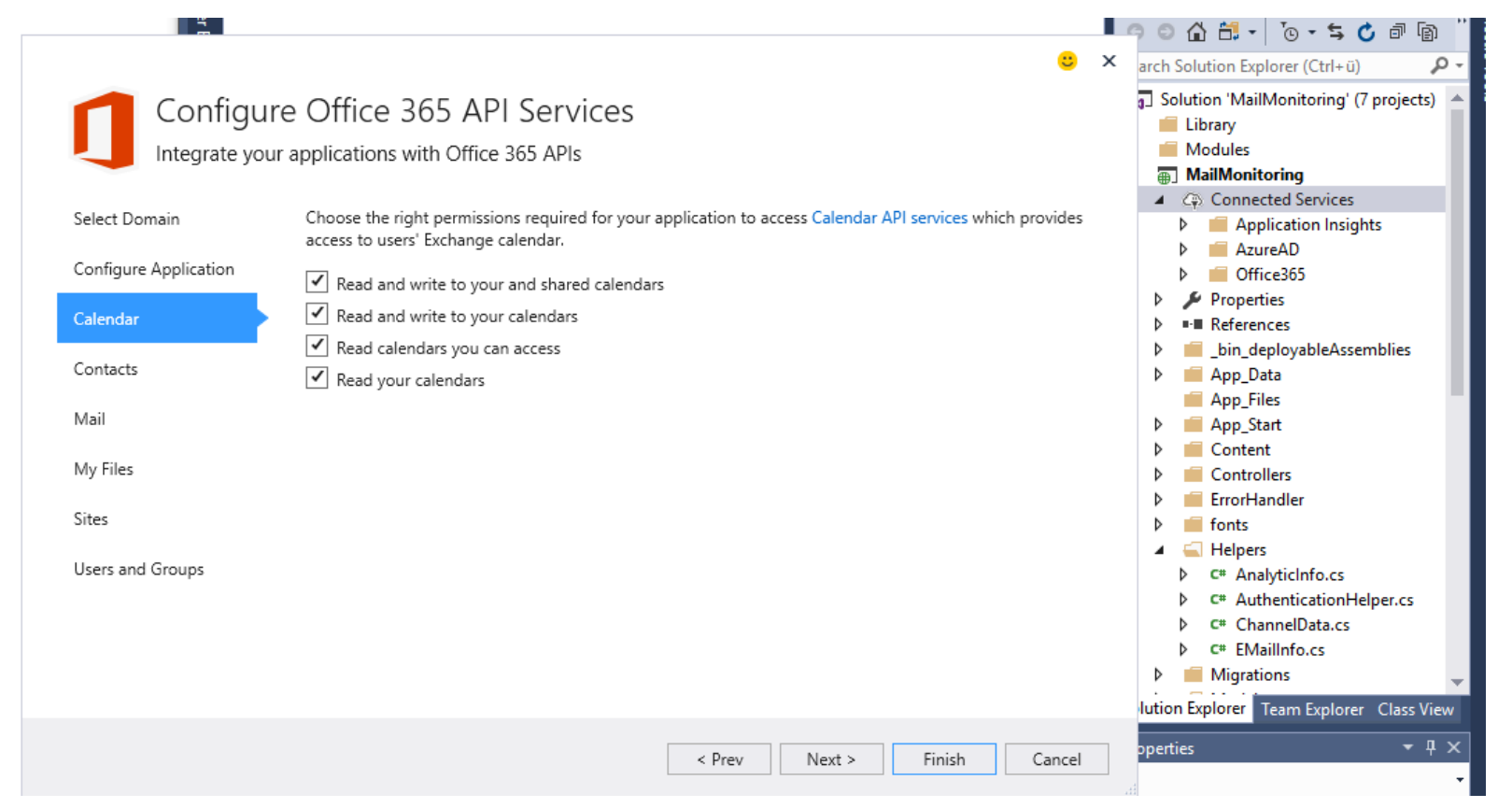

Figure 27: Connected Services

\footnotetext{
${ }^{18}$ http://owin.org/
} 
The implementation requires an Office 365 Developer Tenant and Visual Studio with Microsoft Office 365 API Tools. In the solution explorer, a new Connected Service for Office 365 API is to configure, see a screenshot of this in Figure 27. The credentials for the Office 365 Developer tenant account is to enter and desired permissions are to set. After that the application is registered on Azure Active Directory associated with own Office 365 tenant.

The registration and token management is implemented in the helper class AuthenticationHelper.cs which returned a new OutlookServicesClient access token for secure API calls. A short implementation detail of this helper class is shown in Figure 28.

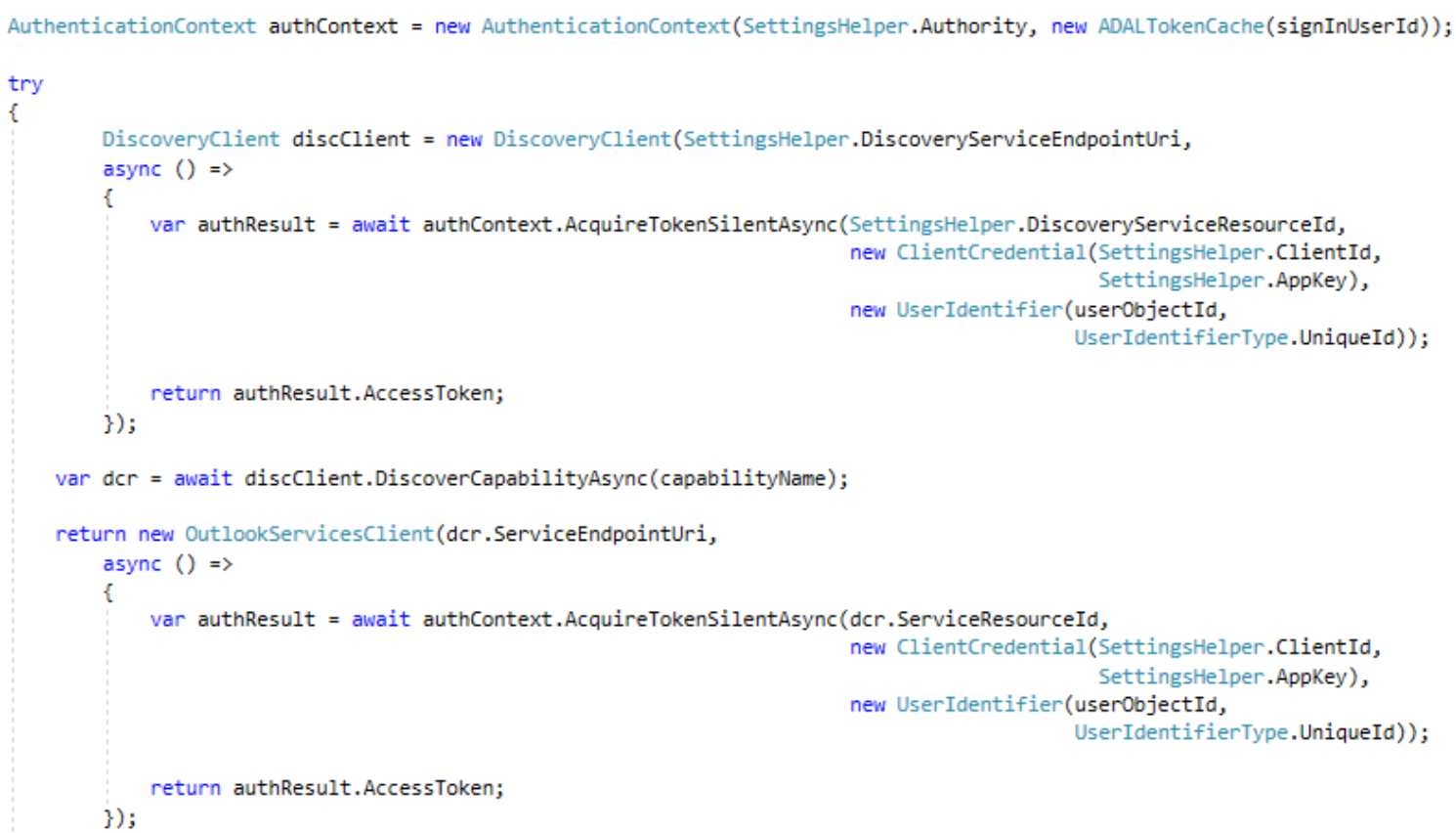

Figure 28: AuthenticationHelper.cs

The bot has a seperated Office365Service class which handels this methods:

- GetCalenderEventAsync: This method gets all events in a certain time span.

- GetCalendarEventDetailsAsync: This method gets the details of an special event.

- AddCalendarEventAsync: This method adds a new event in the calendar.

- UpdateCalendarEventAsync: This method updates a existing event.

- DeleteCalendarEventAsync: This method removes a selected event from the calendar.

- GetEmailMessages: This method gets all Email messages. 
Following the methods GetCalendarEventAsync AddCalendarEventAsync and

GetEmailMessages are described briefly.

\section{GetCalendarEventAsync:}

After getting the access token of the OutlookServiceClient from the AuthenticationHelper the OutlookService Client gets all calendar events between the given timespan. The event information were converted to the own CalendarInfo object and sends a list of them back. The GetCalendarEventsAsync method is shown in Figure 29.

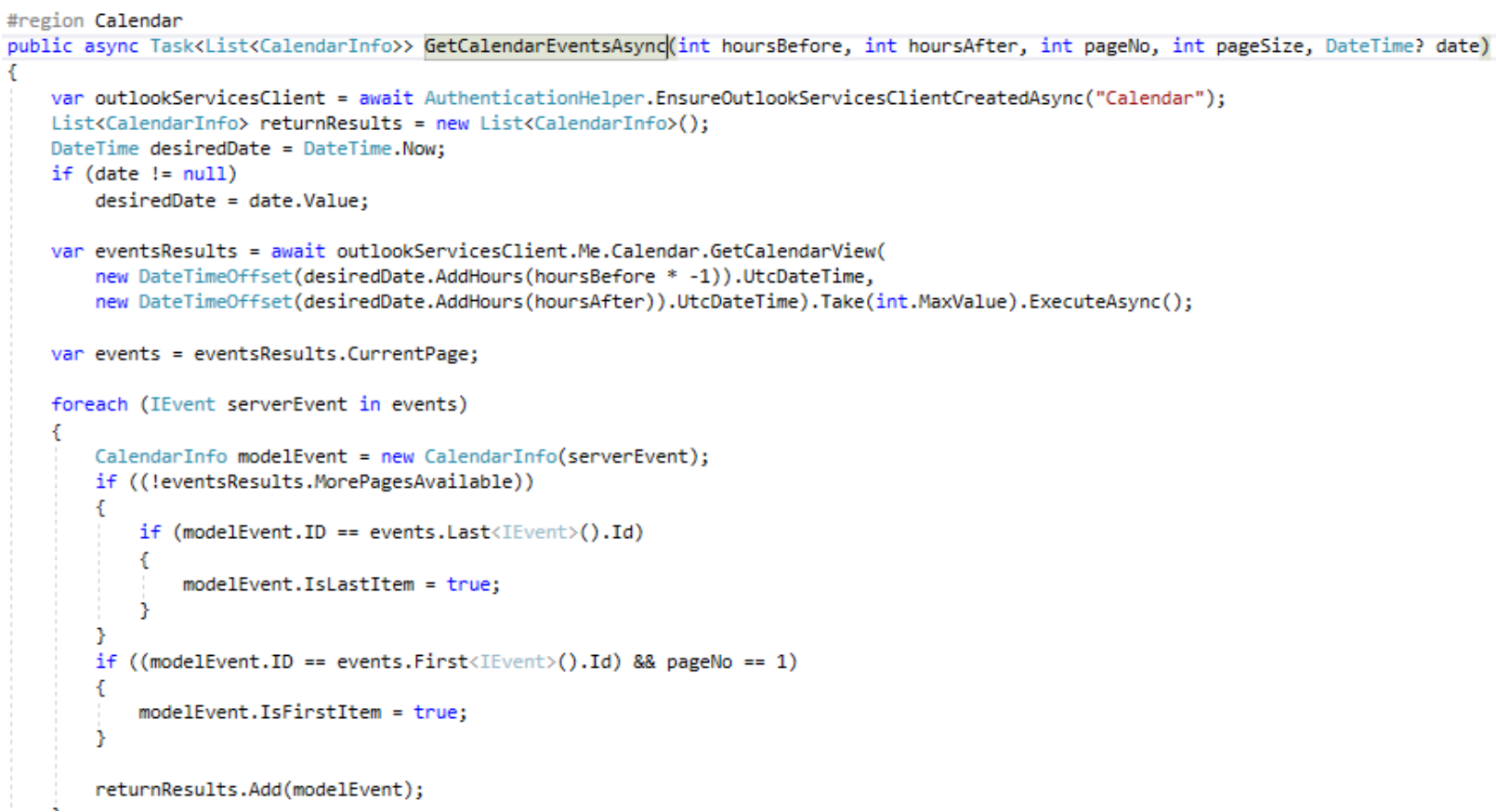

Figure 29: Office365Service GetCalendarEventsAsync method

For the classification, it is necessary to know if there is already a calendar event on the appointment request date and time. Therefore, the bot gets all the events for that request date with a timespan from 2 hours before and 4 hours after by the GetCalenderEventAsync method, shown in Figure 30. If the returned list of CalendarInfos is empty the value of the object FreeAppointment in the decision-making process is set to true. 


\section{AddCalendarEventAsync:}

This method creates a calendar event on the Office 365 account. There are two references to this method in the bot application. Once if the decision-making process was successful the new appointment request will be created and on the other hand on the website it is possible to create a new event by hand.

For creating a new event these parameters are needed: Location-Name, Body-Content, Attendees, Subject, Start-Date and End-Date. First the Event-Object will be created, filled with available information and then after getting the OutlookServiceClient access token the event is added into the event-list under the Office 365 account. The hole method is shown in Figure 31.

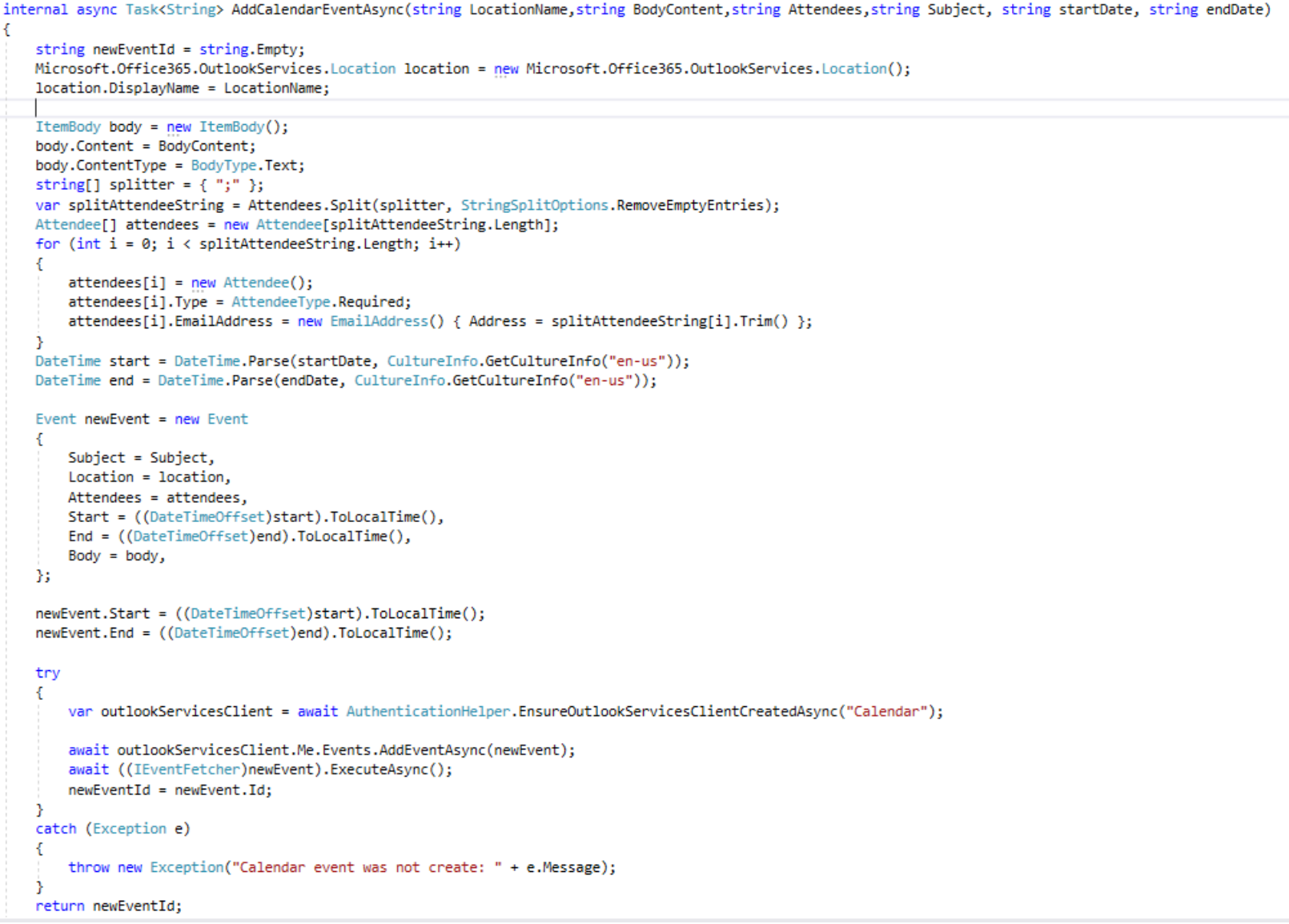

Figure 31: Office365 Service AddCalendarEvent method

\section{GetEmailMessages:}

As it is shown in Figure 32 the method GetEmailMessages gets an OutlookServiceClient access token from the AuthenticationHelpter. With this token, the application calls to the service to 
get all Email messages from the folder "Inbox" limited by a given page size. All returned messages where stored in a List of MailInfo objects and returned them back to the call reference.

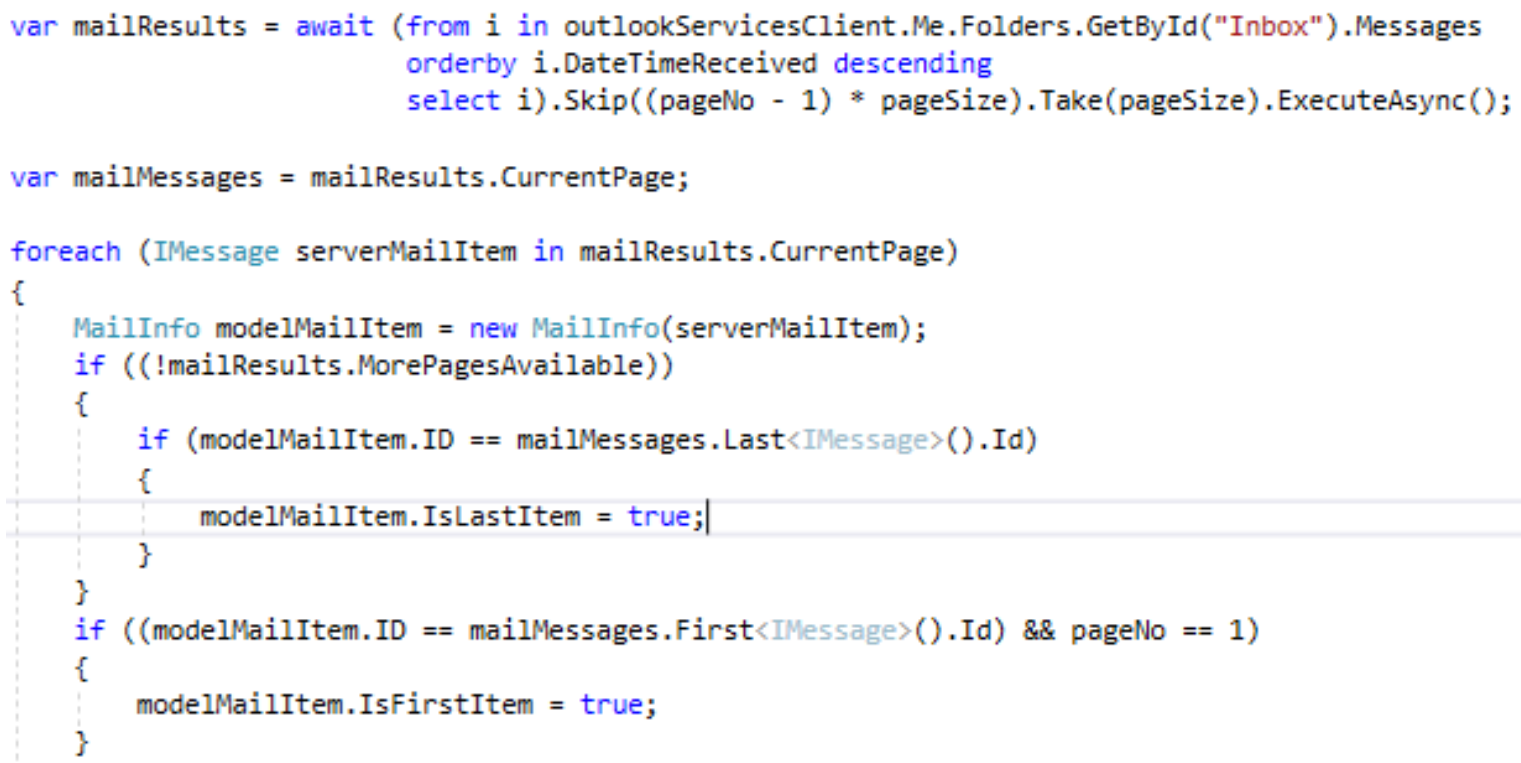

Figure 32: Office365Service GetEmailMessages method 


\subsection{Monitoring and Management Website}

The Website is for monitoring and managing the social bot application. Since the website also access Office 365 account data such as email and calendar, it is necessary to log on in the website with the Office 365 Email address at the beginning. The login page is shown in Figure 33.

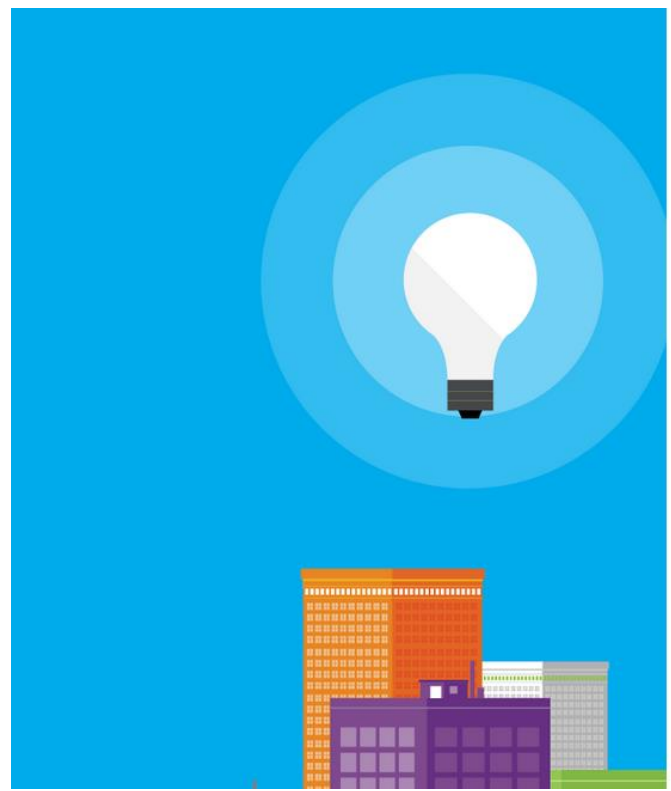

MailMonitoring

商 mailmonitor@waisman...

$\boldsymbol{\oplus}$

Anderes Konto verwenden

Figure 33: Monitoring and Management Website - Login Page

The website serves as a lightweight email client. The bot owner sees his or her last incoming Emails and can easily manage the Office 365 calendar and the decision rules. It means that it is possible to edit, delete or create new calendar events or decision rules.

Including the landing page ("Home"), the website contains six pages:

- Home: All incoming emails from the Office 365 Account and all Office 365 calendar events are listed, shown on Figure 34. 


\section{Mail Monitoring Home Contacts Settings 0365 Calendar DecisionRules ClassificationResult Hello, malimonitior@waismann.eu! Sign out}

Master Thesis: Email Monitoring and Management with Social Bots

Department of Telecooperation - JKU - Manuela Pollak

Office 365 Mails

$\begin{array}{lllll}\begin{array}{l}\text { Sender } \\ \text { manu.pollak@gmail.com }\end{array} & \begin{array}{l}\text { Subject } \\ \text { appointment }\end{array} & \begin{array}{l}\text { Received } \\ 8 / 2 / 2017 \text { 1:43:44 PM }\end{array} & \begin{array}{l}\text { Body } \\ \text { Dear Manuela, we are searching for a new climbing } \\ \text { partner. Date 06. 08 2017 18:30 Would you come with us? } \\ \text { CU }\end{array} & \begin{array}{l}\text { Recipients } \\ \text { mailmonitor@waismann.eu }\end{array} \\ \text { manu.pollak@gmail.com } & \text { appointment } & \text { 8/2/2017 1:08:04 PM } & \begin{array}{l}\text { Servus Manuela, There is a great movie on date 28. 08 } \\ \text { 2017 14:30 in the cinema, Would you come with me? cu }\end{array} & \text { mailmonitor@waismann.eu } \\ \text { manu.pollak@gmail.com } & \text { appointment } & \text { 8/2/2017 1:04:41 PM } & \text { Date 04.08 2017 18:00 sking } & \text { mailmonitor@waismann.eu }\end{array}$

Figure 34: Website - Home page

- Contacts: Email-Address, First Name, Last Name Role Id and Last Action information of the senders are listed.

- Settings: Decision-making Type process (Decision Tree or Naïve Bayes) for the decision-making can be set here.

- O(ffice) 365 Calendar: all Office 365 Calendar information's are listed and can be edit or deleted. Also new Calendar events can be created. The view is shown on Figure 35.

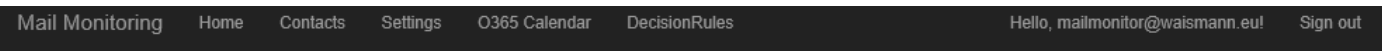

\section{Calendar - Events Refresh}

\begin{tabular}{|c|c|c|c|c|c|}
\hline Subject & LocationSender & $\begin{array}{l}\text { Start } \\
\text { Date }\end{array}$ & $\begin{array}{l}\text { End } \\
\text { Date }\end{array}$ & Body & Action \\
\hline $\begin{array}{l}\text { Indoor } \\
\text { Sport }\end{array}$ & manu.pollak@gmail.com & $\begin{array}{l}07-11- \\
2017 \\
00: 00 \text { AM }\end{array}$ & $\begin{array}{l}\text { 07-11- } \\
2017 \\
\text { 04:00 AM }\end{array}$ & dear,cinema,today,20;0;clock,cu,appointment; & $\begin{array}{l}\text { Edit| } \\
\text { Delete }\end{array}$ \\
\hline Entertainment & manu.pollak@gmail.com & $\begin{array}{l}07-11- \\
2017 \\
16: 00 \text { PM }\end{array}$ & $\begin{array}{l}\text { 07-11- } \\
2017 \\
\text { 20:00 PM }\end{array}$ & tea,time;date;11;07;2017;16;00;come;appointment; & $\begin{array}{l}\text { Edit 1 } \\
\text { Delete }\end{array}$ \\
\hline $\begin{array}{l}\text { Indoor } \\
\text { Sport }\end{array}$ & manu.pollak@gmail.com & $\begin{array}{l}07-12- \\
2017 \\
10: 10 \text { AM }\end{array}$ & $\begin{array}{l}\text { 07-12- } \\
2017 \\
\text { 14:10 PM }\end{array}$ & hi;playing,tennis;date;12;07;2017;10;10; come;appointment; & $\begin{array}{l}\text { Edit | } \\
\text { Delete }\end{array}$ \\
\hline $\begin{array}{l}\text { Indoor } \\
\text { Sport }\end{array}$ & manu.pollak@gmail.com & $\begin{array}{l}07-12- \\
2017 \\
11: 10 \text { AM }\end{array}$ & $\begin{array}{l}07-12- \\
2017 \\
15: 10 \text { PM }\end{array}$ & hi;go;gym;date;12;07;2017;11;10;come;appointment; & $\begin{array}{l}\text { Edit | } \\
\text { Delete }\end{array}$ \\
\hline
\end{tabular}

Figure 35: Website - Office 365 calendar events view page 
- DecisionRules: Decisions respectively the training data are listed in this view page and they can be edit, delete or new one can be created. The view page is shown in Figure 36.

Mail Monitoring Home Contacts Settings 0365 Calendar $\quad$ DecisionRules $\quad$ Hello, mallmonitor@waismann.eu! Sign out

\begin{tabular}{|c|c|c|c|c|c|}
\hline Roleld & FreeAppointment & Type & Weather & Acceptance & \\
\hline 1 & 甲 & Wellness & clear sky & $\square$ & Edit | Delete \\
\hline 1 & ఐ & Wellness & few clouds & $\square$ & Edit | Delete \\
\hline 1 & 四 & Outdoor Sport & light rain & $\square$ & Edit | Delete \\
\hline 1 & $\square$ & Outdoor Sport & rain & $\square$ & Edit | Delete \\
\hline 1 & 四 & Outdoor Sport & few clouds & $\square$ & Edit | Delete \\
\hline 1 & 四 & Outdoor Sport & whatever & $\square$ & Edit | Delete \\
\hline 1 & 甲 & Outdoor Sport & clear sky & $\square$ & Edit | Delete \\
\hline 1 & $\square$ & Outdoor Sport & broken clouds & $\square$ & Edit | Delete \\
\hline 1 & $\square$ & Indoor Sport & whatever & $\square$ & Edit | Delete \\
\hline 1 & $a$ & Inrinnr Cnnert & hrnkon sinuide & $\sqcap$ & Frit I noloto \\
\hline
\end{tabular}

Figure 36: Website - Decisions -Training data view page

By clicking on "Create New"- Link, presented under the heading, a new view start and a new decision can be created. This is shown in Figure 37.

\section{Mail Monitoring Home Contacts Settings 0365 Calendar DecisionRules}

\section{Create}

Decision

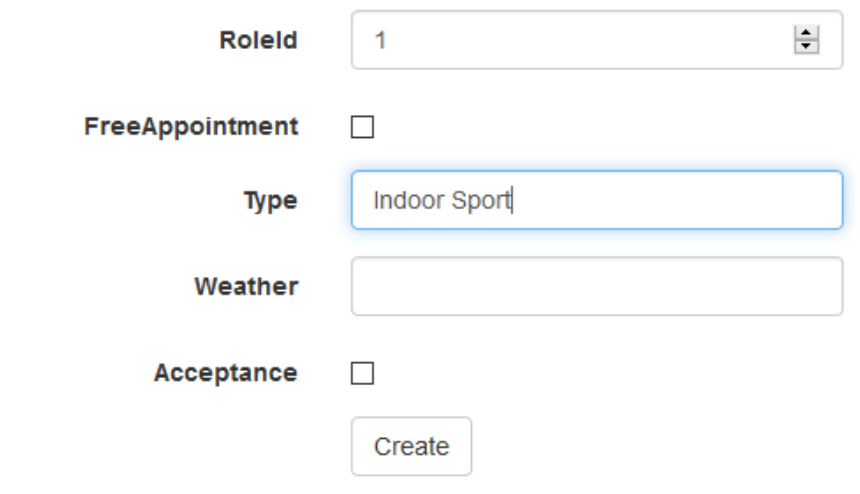

Figure 37: Website - Create new decision view 
- ClassificationResult: For each decision-making process, an entry about the Decision Tree result and the Naïve Bayes result is set in the Table ClassificationResult and listed on this page.

There are two ways to start the decision-making process.

First, if the bot gets an incoming Email appointment request. In this case the Email Address of the sender is stored with the results.

Second, there is the possibility starting sample data by click on the "NewTest"-Link, the sample data are stored in a csv-file located in the App_Files dictionary on the server. Therefore, no Email Address is stored in the database. On Figure 38 a shortcut of this page is shown.

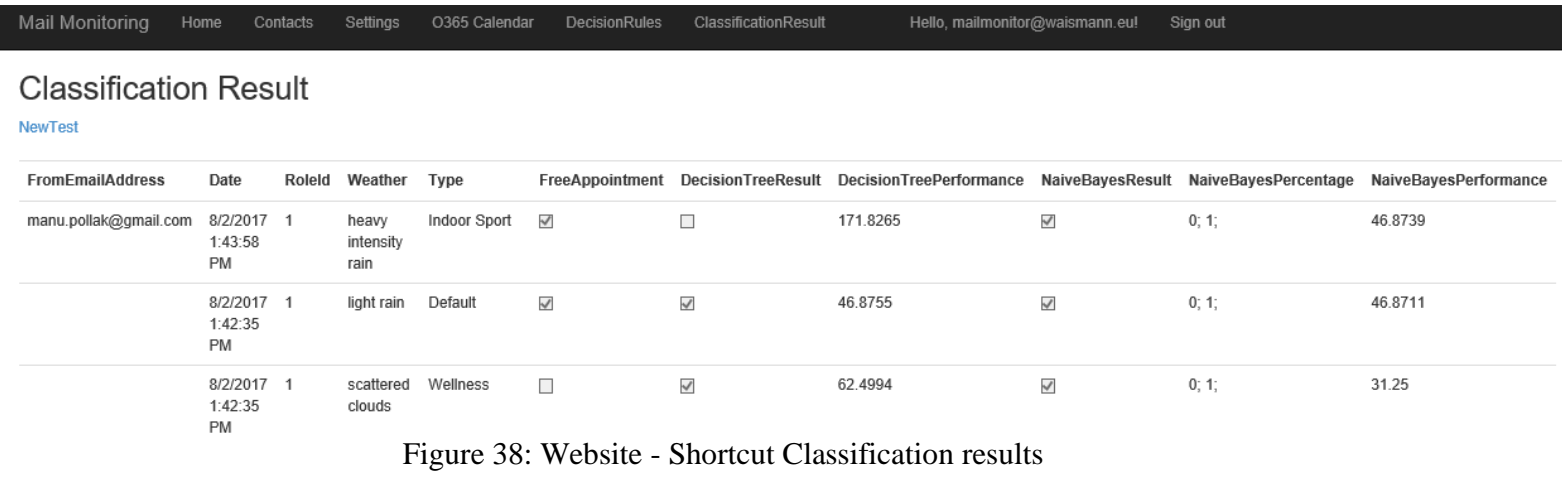




\section{Evaluation}

For the decision-making process two different Classification methods are implemented, The Decision Tree and Naïve Bayes.

Normally the bot application use either the Decision Tree or Naïve Bayes for the decisionmaking process. Which type of classification is to be used can be set on the website. In the evaluation and testing the bot application was modified to uses both decision processes independently of each other on the same set of messages. First the input data will be delivered to the Decision Tree service. Subsequent the Naïve Bayes service started the decision process.

\section{Performance measures for classification}

For binary classifiers, each observation A is mapped to one of two states (Yes or No). The binary response of the classifier can now be correct or false in relation to the (unknown) reality. The results of the classification are summarized in a confusion matrix. This contains the number of all observations in the four possible combinations of classification result and reality. If the classification result is correct, one speaks of "correctly positive" and "correctly negative", depending on which class actually belongs to the observation. If the classifier provides an incorrect answer, then one speaks of a "false positive" or "false negative" decision. Table 1: Evaluation - confusion matrix shows the confusion matrix.

Table 1: Evaluation - confusion matrix

\begin{tabular}{|c|c|c|c|}
\hline & & \multicolumn{2}{|c|}{ Actual value } \\
\hline & & Positive & Negative \\
\hline \multirow{2}{*}{ 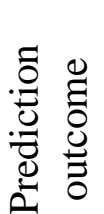 } & Yes & TP & FP \\
\hline & No & FN & $\mathbf{T N}$ \\
\hline
\end{tabular}

- True Positive (TP): Correctly classified as the class of acceptance.

- True Negative (TN): Correctly classified as not the class of acceptance. 
- False Positive (FP): Incorrectly classified as the class of acceptance.

- False Negative (FN): Incorrectly classified as not the class of acceptance.

In order to bring the classifier quality to a more formal and quantitative level, several measures have been defined and calculated in this evaluation on basis of the result data of the sample data.

\section{Accuracy and Error rate}

Accuracy is the percentage of test set sample that are correctly classified by the model.

$$
\text { accuracy }=\frac{T P+T N}{T P+T N+F P+F N}
$$

The error rate: The proportion of incorrectly classified examples, is specified as:

$$
\text { error rate }=\frac{F P+F N}{T P+T N+F P+F N}=1-\text { accuracy }
$$

\section{Precision}

Precision is the ratio of correctly classified that are truly positive.

$$
\text { precision }=\frac{T P}{T P+F P}
$$

\section{Recall}

Recall is the ratio of correctly classified retrieved to the total number of relevant classified.

$$
\text { recall }=\frac{T P}{T P+F N}
$$

\section{F-Measure}

F-Measure is a combination of recall and precision. It is also defined as harmonic mean of precision and recall. 


$$
f-\text { measure }=\frac{2 * \text { recall } * \text { precision }}{\text { recall }+ \text { precision }}
$$

\section{Training data:}

The training data includes 30 different feature combinations, for which it was known whether a term should occur, shown in figure 11. Based on these training data the Decision Tree and Naïve Bayes are able to decide.

\section{Sample data:}

For the evaluation, a sample dataset of 50 different records are stored in a csv-file on the server. A separate service for the evaluation was implemented and can be started on the website, under the menu "Classification Result". The decision-making process results are listed on the same page.

\section{Classification measurement results}

Table 2 listed the sample data with their classification results. Column 1-4 shows the sample data given from a csv-file and the classification results are stored in the next columns:

- Decision Tree Result: In this column, the result of the Decision Tree is placed.

- Naïve Bayes Result: The result of the Naïve Bayes is placed in this column.

- Naïve Bayes \%: In this column, the percentage of the decision is placed.

- Decision Tree Performance /ms: Performance of the Decision Tree is placed in this column. The value is given in milliseconds

- Naïve Bayes Performance /ms: In this column, the performance of the Naïve Bayes is placed in milliseconds.

Table 2: Evaluation - Sample data with Classification results

\begin{tabular}{|c|c|c|c|c|c|c|c|c|}
\hline $\begin{array}{l}\text { Rol } \\
\text { e } \\
\text { Id }\end{array}$ & Weather & Type & $\begin{array}{l}\text { Free } \\
\text { Appointmen } \\
t\end{array}$ & $\begin{array}{l}\text { Decisio } \\
\mathbf{n} \\
\text { Tree } \\
\text { Result }\end{array}$ & $\begin{array}{l}\text { Naive } \\
\text { Bayes } \\
\text { Resul } \\
t\end{array}$ & $\begin{array}{l}\text { Naive } \\
\text { Bayes } \\
\%\end{array}$ & $\begin{array}{l}\text { Decision } \\
\text { Tree } \\
\text { Performanc } \\
\text { e /ms }\end{array}$ & $\begin{array}{l}\text { Naïve } \\
\text { Bayes } \\
\text { Performanc } \\
\text { e /ms }\end{array}$ \\
\hline 1 & $\begin{array}{l}\text { few } \\
\text { clouds }\end{array}$ & Wellness & True & False & True & $\begin{array}{l}0.485 \\
0.515\end{array}$ & 62.4625 & 46.9108 \\
\hline 1 & clear sky & Wellness & True & False & False & $\begin{array}{l}0.586 \\
0.414\end{array}$ & 46.8433 & 62.4984 \\
\hline
\end{tabular}




\begin{tabular}{|c|c|c|c|c|c|c|c|c|}
\hline 1 & $\begin{array}{l}\text { heavy } \\
\text { intensity } \\
\text { rain }\end{array}$ & Wellness & False & False & False & $1 ; 0$ & 78.1234 & 78.1245 \\
\hline 1 & rain & Wellness & True & True & True & $\begin{array}{l}0.414 \\
0.586\end{array}$ & 62.4985 & 62.4993 \\
\hline 1 & light rain & Wellness & True & True & False & $\begin{array}{l}0.739 \\
0.261\end{array}$ & 46.8708 & 62.5003 \\
\hline 1 & $\begin{array}{l}\text { broken } \\
\text { clouds }\end{array}$ & Wellness & False & False & False & $1 ; 0$ & 62.4992 & 62.5162 \\
\hline 1 & $\begin{array}{l}\text { few } \\
\text { clouds }\end{array}$ & Wellness & False & False & False & $1 ; 0$ & 62.5104 & 46.881 \\
\hline 1 & whatever & Wellness & True & False & False & $1 ; 0$ & 46.8675 & 46.878 \\
\hline 1 & $\begin{array}{l}\text { moderate } \\
\text { rain }\end{array}$ & Wellness & False & False & False & $1 ; 0$ & 109.374 & 62.4924 \\
\hline 1 & $\begin{array}{l}\text { scattered } \\
\text { clouds }\end{array}$ & Wellness & False & False & False & $1 ; 0$ & 46.8732 & 46.8739 \\
\hline 1 & $\begin{array}{l}\text { broken } \\
\text { clouds }\end{array}$ & $\begin{array}{l}\text { Outdoor } \\
\text { Sport }\end{array}$ & True & False & False & $1 ; 0$ & 46.8719 & 46.866 \\
\hline 1 & rain & $\begin{array}{l}\text { Outdoor } \\
\text { Sport }\end{array}$ & True & False & True & $\begin{array}{l}0.282 \\
0.718\end{array}$ & 62.5044 & 62.5035 \\
\hline 1 & $\begin{array}{l}\text { scattered } \\
\text { clouds }\end{array}$ & $\begin{array}{l}\text { Outdoor } \\
\text { Sport }\end{array}$ & True & True & True & $\begin{array}{l}0.440 \\
0.560\end{array}$ & 62.5002 & 62.4906 \\
\hline 1 & light rain & $\begin{array}{l}\text { Outdoor } \\
\text { Sport }\end{array}$ & False & False & False & $1 ; 0$ & 46.8796 & 93.756 \\
\hline 1 & clear sky & $\begin{array}{l}\text { Outdoor } \\
\text { Sport }\end{array}$ & True & True & True & $\begin{array}{l}0.440 \\
0.560\end{array}$ & 46.8662 & 46.8744 \\
\hline 1 & $\begin{array}{l}\text { few } \\
\text { clouds }\end{array}$ & $\begin{array}{l}\text { Outdoor } \\
\text { Sport }\end{array}$ & True & True & True & $\begin{array}{l}0.344 ; \\
0.656 ;\end{array}$ & 62.499 & 62.4994 \\
\hline 1 & $\begin{array}{l}\text { heavy } \\
\text { intensity } \\
\text { rain }\end{array}$ & $\begin{array}{l}\text { Outdoor } \\
\text { Sport }\end{array}$ & False & False & False & $1 ; 0$ & 62.5149 & 62.4976 \\
\hline 1 & whatever & $\begin{array}{l}\text { Outdoor } \\
\text { Sport }\end{array}$ & True & False & False & $1 ; 0$ & 46.873 & 46.8616 \\
\hline 1 & $\begin{array}{l}\text { moderate } \\
\text { rain }\end{array}$ & $\begin{array}{l}\text { Outdoor } \\
\text { Sport }\end{array}$ & False & False & False & $1 ; 0$ & 46.8769 & 62.4913 \\
\hline 1 & clear sky & $\begin{array}{l}\text { Outdoor } \\
\text { Sport }\end{array}$ & False & False & False & $1 ; 0$ & 62.4875 & 62.4984 \\
\hline 1 & $\begin{array}{l}\text { heavy } \\
\text { intensity } \\
\text { rain }\end{array}$ & Indoor Sport & False & False & False & $1 ; 0$ & 46.8758 & 46.8849 \\
\hline 1 & rain & Indoor Sport & True & True & True & $\begin{array}{l}0.150 \\
0.840\end{array}$ & 62.48 & 46.8745 \\
\hline 1 & whatever & Indoor Sport & True & False & False & $1 ; 0$ & 46.8836 & 46.8205 \\
\hline 1 & $\begin{array}{l}\text { scattered } \\
\text { clouds }\end{array}$ & Indoor Sport & False & False & False & $1 ; 0$ & 46.9107 & 46.8391 \\
\hline 1 & $\begin{array}{l}\text { few } \\
\text { clouds }\end{array}$ & Indoor Sport & True & True & True & $\begin{array}{l}0.190 \\
0.810\end{array}$ & 62.49 & 93.7436 \\
\hline 1 & $\begin{array}{l}\text { moderate } \\
\text { rain }\end{array}$ & Indoor Sport & True & True & True & $\begin{array}{l}0.261 \\
0.739\end{array}$ & 46.8955 & 46.8696 \\
\hline
\end{tabular}




\begin{tabular}{|c|c|c|c|c|c|c|c|c|}
\hline 1 & $\begin{array}{l}\text { broken } \\
\text { clouds }\end{array}$ & Indoor Sport & False & False & False & $1 ; 0$ & 46.8746 & 62.4661 \\
\hline 1 & clear sky & Indoor Sport & False & False & False & $1 ; 0$ & 46.8625 & 46.9118 \\
\hline 1 & light rain & Indoor Sport & False & False & False & $1 ; 0$ & 46.8373 & 84.0537 \\
\hline 1 & light rain & Indoor Sport & True & True & True & $\begin{array}{l}0.414 \\
0.586\end{array}$ & 46.8746 & 46.8792 \\
\hline 1 & clear sky & $\begin{array}{l}\text { Entertainmen } \\
\mathrm{t}\end{array}$ & True & False & True & $\begin{array}{l}0.414 \\
0.586\end{array}$ & 62.4899 & 46.8781 \\
\hline 1 & rain & $\begin{array}{l}\text { Entertainmen } \\
\mathrm{t}\end{array}$ & False & False & False & $1 ; 0$ & 46.8753 & 62.5378 \\
\hline 1 & clear sky & $\begin{array}{l}\text { Entertainmen } \\
\mathrm{t}\end{array}$ & True & False & True & $\begin{array}{l}0.414 \\
0.586\end{array}$ & 46.8762 & 62.4989 \\
\hline 1 & $\begin{array}{l}\text { scattered } \\
\text { clouds }\end{array}$ & $\begin{array}{l}\text { Entertainmen } \\
\mathrm{t}\end{array}$ & False & False & False & $1 ; 0$ & 62.4979 & 62.502 \\
\hline 1 & light rain & $\begin{array}{l}\text { Entertainmen } \\
\mathrm{t}\end{array}$ & True & True & False & $\begin{array}{l}0.586 \\
0.414\end{array}$ & 171.865 & 62.4612 \\
\hline 1 & $\begin{array}{l}\text { few } \\
\text { clouds }\end{array}$ & $\begin{array}{l}\text { Entertainmen } \\
\mathrm{t}\end{array}$ & False & False & False & $1 ; 0$ & 46.8949 & 46.833 \\
\hline 1 & whatever & $\begin{array}{l}\text { Entertainmen } \\
\mathrm{t}\end{array}$ & False & False & False & $1 ; 0$ & 62.5003 & 78.1309 \\
\hline 1 & $\begin{array}{l}\text { moderate } \\
\text { rain }\end{array}$ & $\begin{array}{l}\text { Entertainmen } \\
\mathrm{t}\end{array}$ & True & True & True & $\begin{array}{l}0.414 \\
0.586\end{array}$ & 62.5568 & 62.4638 \\
\hline 1 & $\begin{array}{l}\text { scattered } \\
\text { clouds }\end{array}$ & $\begin{array}{l}\text { Entertainmen } \\
\mathrm{t}\end{array}$ & True & True & True & $\begin{array}{l}0.414 \\
0.586\end{array}$ & 46.8369 & 62.5071 \\
\hline 1 & $\begin{array}{l}\text { heavy } \\
\text { intensity } \\
\text { rain }\end{array}$ & $\begin{array}{l}\text { Entertainmen } \\
\mathrm{t}\end{array}$ & True & True & True & $\begin{array}{l}0.190 \\
0.810\end{array}$ & 46.8714 & 46.8653 \\
\hline 1 & clear sky & Default & True & True & True & $\begin{array}{l}0.239 \\
0.761\end{array}$ & 78.125 & 78.1228 \\
\hline 1 & rain & Default & False & False & False & $1 ; 0$ & 62.4583 & 46.8747 \\
\hline 1 & $\begin{array}{l}\text { heavy } \\
\text { intensity } \\
\text { rain }\end{array}$ & Default & True & True & True & $\begin{array}{l}0.095 \\
0.905\end{array}$ & 46.8752 & 62.4967 \\
\hline 1 & $\begin{array}{l}\text { few } \\
\text { clouds }\end{array}$ & Default & False & False & False & $1 ; 0$ & 62.493 & 46.9098 \\
\hline 1 & $\begin{array}{l}\text { scattered } \\
\text { clouds }\end{array}$ & Default & True & True & True & $\begin{array}{l}0.239 \\
0.761\end{array}$ & 46.8753 & 46.8744 \\
\hline 1 & $\begin{array}{l}\text { moderate } \\
\text { rain }\end{array}$ & Default & False & False & False & $1 ; 0$ & 46.9091 & 46.9104 \\
\hline 1 & $\begin{array}{l}\text { few } \\
\text { clouds }\end{array}$ & Default & True & True & True & $\begin{array}{l}0.173 \\
0.827\end{array}$ & 62.5364 & 46.8707 \\
\hline 1 & whatever & Default & False & False & False & $1 ; 0$ & 46.8386 & 46.9111 \\
\hline 1 & $\begin{array}{l}\text { broken } \\
\text { clouds }\end{array}$ & Default & False & False & False & $1 ; 0$ & 109.3742 & 46.9139 \\
\hline 1 & light rain & Default & True & True & True & $\begin{array}{l}0.386 \\
0.614\end{array}$ & 62.4686 & 46.9103 \\
\hline
\end{tabular}




\begin{tabular}{|c|c|c|c|}
\hline & & Act & alue \\
\hline & & Positive & Negative \\
\hline. & Yes & 17 & 0 \\
\hline 总 & No & 3 & 30 \\
\hline
\end{tabular}

Table 3 shows result of the Decision Tree process placed in the confusion matrix and in Table 4 the result of the Naïve Bayes process is shown placed in the confusion matrix. It can be seen that the Decision Tree has no FP and only three times the result was FN. However, the Naïve Bayes has summarized nine false defined results.

Table 4: Evaluation - confusion matrix result Naive Bayes

\begin{tabular}{|c|c|c|c|}
\hline & & & alue \\
\hline & & Positive & Negative \\
\hline$\stackrel{0}{\Xi}$ & Yes & 15 & 4 \\
\hline 吾 & No & 5 & 26 \\
\hline
\end{tabular}

In Table 5 the measures result of both implemented classification technologies, Decision Tree and Naïve Bayes, are shown.

Table 5: Evaluation - Classification measures

\begin{tabular}{|l|l|l|}
\cline { 2 - 3 } \multicolumn{1}{c|}{} & Decision Tree & Naïve Bayes \\
\hline Accuracy & $94 \%$ & $82 \%$ \\
\hline Error rate & $6 \%$ & $18 \%$ \\
\hline
\end{tabular}




\begin{tabular}{|l|l|l|}
\hline Precision & $100 \%$ & $79 \%$ \\
\hline Recall & $85 \%$ & $75 \%$ \\
\hline F-measure & $92 \%$ & $77 \%$ \\
\hline
\end{tabular}

\section{Accuracy \& Error rate}

Accuracy is the most performance measure and it is the ratio of correctly predicted observations. The Decision Tree has an accuracy of $94 \%$ and so an error rate from only $6 \%$. Compared to the Naïve Bayes, with an accuracy of $82 \%$ and an error rate of $18 \%$, the Decision Tree is more correct by decision-making.

\section{Precision}

Precision shows the ratio of correct positive observations. Because of the zero FP rate from the Decision Tree the precision is complete $100 \%$ for the Decision Tree where Naïve Bayes only has a precision of $79 \%$.

\section{Recall}

Recall is also knowns as sensitivity and it's the ratio of correctly predicted positive events. Also in this measurement, the Decision Tree better cut of with $85 \%$. Naïve Bayes only has a recall of $75 \%$

\section{F-measure}

F-measure or also called F1 score or just F score conveys the balance between the precision and the recall. The F-measure rate of the Decision Tree is very high with $92 \%$ where Naïve Bayes only reach $77 \%$.

The measures show clear that the Decision Tree is quite effective compared to the Naïve Bayes for the classification of the appointment requests. Even on performance, shown in Table 4, the Decision Tree is significantly faster during the decision-making process. 


\section{Conclusions and Future Work}

The main question of this master thesis was:

Is there a possibility where social bots can help us with our daily Email flood?

Yes, there is a possibility and in combination with Artificial Intelligence, short AI, the possibilities are endless. The combination of social bots and AI is a very interesting and complex subject area and full of possibilities.

The master thesis is about automatizing Email appointment request. Therefore, different classification techniques were discussed and the Decision Tree and Naïve Bayes were implemented. The evaluation has shown that the Decision Tree is more accurate and less susceptible for errors.

The Microsoft Bot Framework is a high-potential and powerful technology and it can be easily combined with different other frameworks or Restful webservices. Especially the integration of Office 365 and Accord.net gives the bot application the necessary tools to get the social bot to a powerful Email client, even if the development is still far away.

The bot application can be used for answering emails in general and not only for appointment requests so this chapter discusses potential extensions to the bot application. These are functionalities that are not included in this version.

\section{Artificial Intelligence:}

In future work methods, decision-making process with clustering and the Support Vector Machine of Classification will be interesting. Especially in comparison to the Decision Tree.

\section{Decision-making process}

The assignment to types and the combination between types and weather has to be defined more exactly. After that the decision rules must be extended and refined.

This master thesis has started with a rather narrow set of email in the private sector, next steps are the extension of rules to be more suitable in a working or business context. 


\section{Website and Office 365}

The website is the user interface for the Bot-Email client. The most important next step is the integration of Office 365 contacts with the bot application. Because with the usage of the Office 365 contacts the bot owner has more benefit than the integrated contact in the database. In Office 365 contacts data new fields can be defined used for the priority of the sender emails requests. 


\section{References}

[ACC17] Accord-Framework.net, $\quad$ NaiveBayes, $\underline{\text { http://accord- }}$ framework.net/docs/html/T_Accord_MachineLearning_Bayes_NaiveBayes_1.htm, last visited on July $10^{\text {th }} 2017$.

[AuM08] Auria L., Moro R. A., Support Vector Machines (SVM) as a Technique for Solvency Analysis, Deutsches Institut für Wirtschaftsforschung Berlin, August 2008

[BES16] Bessi Alessandro, Ferrara Emilio, Social bots distort the 2016 U.S. Presidential election online discussion, Peer-Reviewed Journal on the Internet, First Monday, Volume 21, Number 11 . November $7^{\text {th }} 2016$

[BMB11] Boshmaf Y., Muslukhov I., Beznosov K., Ripeanu M., The Socialbot Network: When Bots Socialize for Fame and Money, University of British Columbia Vancouver, Canada, September $27^{\text {th }} 2011$

[BRU16] Brükler P, Social Bots: die virtuelle Pest des 21. Jahrhunderts, https://medium.com/deutsch/social-bots-die-virtuelle-pest-des-21-jahrhunderts-

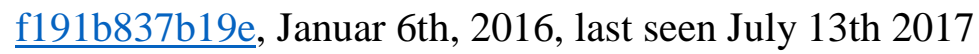

[CHW72] Colby K. M., Hilf F. D, Weber S., Kraemer H.C., Turing-like Indistinguishability Tests for the Validation of a Computer Simulation of Paranoid Processes', Artificial Intelligence 3, pp. 199-222., January 1972

[COR95] Cortes C., Vapnik V. Support-vector network. Machine Learning. 1995.

[DAB08] De Angeli A., Brahnam S., I hate you! Disinhibition with virtual partners, Interacting with Computers 20 (2008) 302-310, February 13 ${ }^{\text {th }} 2008$

[Der10] Deryugina O. V., Chatterbots, Scientific and Technical Information Processing, 2010, Vol. 37, No. 2, pp. 143-147., March $17^{\text {th }} 2010$

[DVF16] Davis C. A., Varol O., Ferrara E., Flammini A., Menczer F., BotOrNot: A System to Evaluate Social Bots, International World Wide Web Conferences Steering Committee, April 2016 
[DYK13] Dykstra Tom, Implementing the Repository and Unit of Work Patterns in a ASP.NET MVC Application, https://docs.microsoft.com/en-us/aspnet/mvc/overview/olderversions/getting-started-with-ef-5-using-mvc-4/implementing-the-repository-and-unit-ofwork-patterns-in-an-asp-net-mvc-application, July $30^{\text {th }} 2013$, last seen July $10^{\text {th }} 2017$

[Fer10] Ferrucci D., Build Watson: an overview of DeepQA for Jeopardy! Challenge, 19th International Conference on Parallel Architectures and Compilation Techniques (PACT), 2010

[Fer15] Emilio Ferrara, Onur Varol, Clayton Davis, Filippo Menczer, and Alessandro Flammini. 2015. The Rise of Social Bots. (June 26th 2015)

[FHM15] Forelle M., Howard P., Monroy-Hernández A., Svage S., Political Bots and the Manipulation of Public Opinion in Venezuela, July $26^{\text {th }} 2015$

[FPS96] Fayyad U., Piatetsky-Shapiro G., Smyth P. "From Data Mining to Knowledge Discovery in Databases" (PDF). 1996.

[FUZ16] Fu Zhe, Zhang Hao. Application of Naive Bayes Classifier in Stampede Risk Earlywarning of Large-scale Activities. International Conference on Industrial Informatics. 2016

[GeB16] Gehl R. W., Bakardjiva M., Socialbots and Their Friends: Digital Media and the Automation of Sociality, Digital Media and Automation of Sociality, December 2016

[HTF09] Trevor Hastie, Robert Tibshirani, Jerome Friedman. The Elements of Statistical Learning: Data Mining Inference and Prediction. Second Edition. Springer; 2009. ISBN 9780-387-84857-0.

[LeY09] Lee C-H., Yang H-C, Construction of supervised and unsupervised learning systems for multilingual text categorization, Expert Systems with Applications 36 (2009) 2400-2410, 2009

[LUG08] Luger G. F.. Artificial Intelligence: Structures and Strategies for Complex Problem Solving. Addison Wesley Pub Co Inc; sixth edition. 2008

[MAU94] Mauldin M. L., Chatterbots, tinymuds, and the turing test: entering the loebner prize com-petition, In Proceedings of the twelfth national conference on Artificial intelligence (vol. 
1), AAAI '94, pages 16\{21, Menlo Park, CA, USA,1994. American Association for Artificial Intelligence.

[Mai10] Maimon O., Rokach L., Data Mining and Knowledge Discovery Handbook, Second Edition, Springer New York Dordrecht Heidelberg London, 2010

[Mill09] Ian Millington, John Funge, editors. Artificial intelligence for games, second edition, Burlington: Morgan Kaufmann Publishers, 2009. ISBN 978-0-12-374731-0.

[New16] Newman J., How the new, improved chatbots rewrite 50 years of bot history, https://www.fastcompany.com/3059439/why-the-new-chatbot-invasion-is-so-different-fromits-predecessors, February $5^{\text {th }} 2016$, last seen on July $11^{\text {th }} 2017$

[PAT12] Patel N, Upadhyay S. Study of various decision tree pruning methods with their empirical comparison in WEKA. International Journal of Computer Applications (0975 -8887) Volume 60-No.12, December 2012

[Per16] Perez S., Microsoft silences its new A.I. bot Tay, after Twitter users teach it racism, https://techcrunch.com/2016/03/24/microsoft-silences-its-new-a-i-bot-tay-after-twitter-usersteach-it-racism/ , March $24^{\text {th }} 2016$

[PSD17] Perlman J. M., Standefer R., Delimarsky D.. How the Bot Framework works. https://docs.microsoft.com/en-us/bot-framework/overview-how-bot-framework-works, last visited on May 20th 2017

[Pol17] Pollak M.. Social Bots with Microsoft Bot Framework, JKU Department of Telecooperation, February 2017

[QUI92] Quinlan J.R. C4.5: Programs for Machine Learning. Morgan Kaufman Publishers, 1993

[ROK08] Rokach, L., Maimon O. Data Mining with Decision Trees: Theory and Applications. World Scientific Pub Co., 32008.

[SBW99] Stern M. K., Beck J. E., Woolf B. P., Naïve Bayes Classifiers for User Modeling, Computer Science Department University of Massachusetts, http://citeseerx.ist.psu.edu/viewdoc/summary?doi=10.1.1.118.979, 1999 
[SLD16] scikit-learn developers (BSD License). Naïve Bayes. http://scikitlearn.org/stable/modules/naive_bayes.html, last visited on May 10th 2017

[Son15] Yan-yan Song, Ying LU. Decision tree methods: applications for classification and prediction. Shanghai Archives of Psychiatry, 2015, Vol. 27, No. 2

[Sou12] CR Souza. Decision Trees in C\# 0. http://crsouza.com/2012/01/04/decision-trees-inc/, last visited on March 20th 2017.

[STA17] Standefer R., Delimarsky D.. Bot Framework Overview. https://docs.microsoft.com/en-us/botframework/overview-introduction-bot-framework, last visited on May 22th 2017

[STO16] Lee Stott. What is Microsoft Bot Framework Overview. April 52016. https://blogs.msdn.microsoft.com/uk_faculty_connection/2016/04/05/what-is-microsoft-botframework-overview/. [15. 05. 2017].

[SGS17] Schmidt M., Gerdt A., Skoruppa P., Google Assistant: Neue Funktionen ... , http://www.computerbild.de/artikel/cb-News-App-Check-Google-Now-Google-Assistant16367107.html, Mai 5th 2017.

[TSK05] Pang-Ning Tan, Michael Steinbach, Vipin Kumar. Introduction to Data Mining. Addision-Wesley Longman Publishing Co., Inc. Boston, 2005. ISBN 0321321367

[Tur50] Alan M Turing. 1950. Computing machinery and intelligence. Mind 49, 236 (1950), 433-460.

[Vin16] James Vincent, Microsoft's wild vision for the future puts conversations at the heart of computing, March 30 2016, http://www.theverge.com/2016/3/30/11331388/microsoftchatbots-ai-build, [27. 11. 2016].

[Wal17] Wallace R. S., The Anatomy of A.L.I.C.E. Retrieved , A.L.I.C.E. Artificial Intelligence Foundation, Inc., http://www.alicebot.org/anatomy.html, last seen on July $20^{\text {th }}$ 2017

[Wei66] Weizenbaum, J. ELIZA--A Computer Program For the Study of Natural Language Communication Between Man and Machine. Communication ACM 9, 1 (January 1966), 36- 


\section{Curriculum Vitae}

\section{Personal Details}

- Name: Manuela Pollak

- E-Mail: $\quad$ manuela@waismann.eu

\section{Work Experience}

$2010-2017$

creative BITS OG

○ Web programmer

○ Project management

$2001-2005$

EDV-Hofer

○ Software developer

\section{Education}

$2011-$

$2005-2010$

$1996-2001$

Organisation)
Master Computer Science, JKU Linz

Bachelorstudium Informatik JKU Linz

Handelsakademie in Kirchdorf/Krems Matura (Informatik und 


\section{Statutory declaration}

I hereby declare that the thesis submitted is my own unaided work, that I have not used other than the sources indicated, and that all direct and indirect sources are acknowledged as references.

This printed thesis is identical with the electronic version submitted.

Bad Wimsbach-Neydharting, August 2017

Manuela Pollak

\section{Eidesstattliche Erklärung}

Ich erkläre an Eides statt, dass ich die vorliegende Diplom- bzw. Magisterarbeit selbstständig und ohne fremde Hilfe verfasst, andere als die angegebenen Quellen und Hilfsmittel nicht benutzt bzw. die wörtlich oder sinngemäß entnommenen Stellen als solche kenntlich gemacht habe.

Die vorliegende Masterarbeit ist mit dem elektronisch übermittelten Textdokument identisch.

Bad Wimsbach-Neydharting, August 2017

Manuela Pollak 